\title{
Calcul de la cheminée à étranglement de la chute Isère-Arc
}

\section{Calculation of the Isère-Arc restricted orifice surge tank}

\author{
PAR M. BOUVARD ET J. MOLBERT \\ INGBNLETS A L'BLEGHICITE DE FOANGE (R.E.H. ALPLS 11)
}

\begin{abstract}
Le choix d'une cheminée à étranglement et à chumbres d'expansion supérieures, compte tenu des dispositions ef des caractiristiques hydrauliques, d'une part, des problemes de Génie Cüvil, d'autre part. - Calcul de la cheminée (puits, stranglement ef chambres d'expansion) en fonction des conditions d'exploitation de la centrale et de la sécurité des ouvrages. - Adoption d'un puils antibétier pour protéger, contre les surpressions dues à l'étranglement, le. troncon en bélon armé des ouvrages d'amenée. - Calenl des carteféristiques et de l'implantation de ce puits. - Etude du comportement des ouvrages dans un certain nombre de as de fonctionnement.
\end{abstract}

\begin{abstract}
Choice of a restricted orifice surge lank with upper expansion chambers, account being taken of the layout and hydranlic characteristics of the structures and of the civil engineering problems.-Calculation of the surge lank (well, orifice and expansion chambers? as a function of the working conditions of the power plant and the safety of the structures.-Adoption of an anti-waterhammer well for protection of the length of reinforeed concrete pipe of the intake structures against pressure surges resulting from the orifice. Calculation of the characteristics and position of this well-Study of the behaviour of the struclures in a certuin number of operational cases.
\end{abstract}

L'aménagement d'Isère-Arc utilise les caux de I'Isc̀re captées en aval de Moutiers et les restitue dans l'Arc au voisinage d'Aigueblanche, sous une chute de $150 \mathrm{~m}$; le débil maximum dérivé est de $100 \mathrm{~m} \mathrm{~m}^{3} / \mathrm{s}$.

\section{I. - GENERALITES}

\section{$1^{\circ}$ Description des ouvrages}

Nous allons décrire brièvement différentes parties de cette chute en détaillant certains points pouvant avoir de l'influence sur le projet de cheminée d'équilibre.

Le harrage et la prise d'eau sont placés au lieu dit «Les Echelles d'Annibal » à l'extrémité aval du défilé précédant la localité d'Aigueblanche.

Le barrage est du type poids; le niveau normal ctant fixć à la cote 471 , le volume de la retenue est de $400.000 \mathrm{~m}^{\circ}$ environ jusqu'au niveau minimum prévu à la cote 466 ; cette rete- nue pourra être utilisée pour compenser on recaler les pointes de débit des usines utilisant les retenues amont, actuellement Tignes et ultẻrieurement Champagny. Il n'est cependant pas exclu que l'on utilise également comme réserve la galerie d'amenée dont la capacité est voisine de celle de la retenue.

La dérivation en charge comporte plusieurs parties :

a) Un tronçon de conduite en béton armé de $6,40 \mathrm{~m}$ de diametre $\left(32 \mathrm{~m}^{2}\right)$ et $2.600 \mathrm{~m}$ de longueur environ; la charge sur cette conduite varie de 10 à $18 \mathrm{~m}$, mais nous signalons, ef cette 
considération est importante, que le prix croit très vite en fonction de la pression;

b) On entre ensuite en souterrain au lieu dit l'Etrat dans du terrain consitué par du schiste du Lias. Ce rocher est de bonne qualité quoique la couverture soit relativement faible; la section reste circulairc, de $\varnothing 6,40 \mathrm{~m}$;

c) Après une courte traversée dans le trias de Ia Léchère, sur une centaine de mètres environ, la galerie entre dans le cristallin qu'elle ne quitte plus jusqu'à la fenêtre de Notre-Dame-deBriançon qui constitue la dernière attaque côté Isère; la section de $32 \mathrm{~m}^{2}$ est alors en fer à cheval;

d) Enfin, le massif montagneux séparant l'Isère de l'Arc est traversé par un tronçon sans fenêtre de $11 \mathrm{~km}$ de long, tout entier situé dans le cristallin, sauf quelques passages de courte longueur prévus dans le trias. Le terrain est, en général, bon, mais il existe cerlains tronçons où le rocher est plus mauvais et nécessite un revêtement très sérieux. C'est à l'aval de ce troncon, côté Arc, que se trouve placée la cheminée d'équilibre souterraine.

La conduite forcée est constituée par deux conduites indépendantes souterraines et blindées, de $3,70 \mathrm{~m}$ de diamètre, précédées de robinets papillon à fermeture automatique.

La centrale est souterraine; clle comporle quatre groupes Francis à axe vertical turbinant chacun $25 \mathrm{~m}^{3} / \mathrm{s}$; chaque groupe sera relic à un transformateur, mais l'énergie sera évacuée par deux lignes indépendantes à 150.000 Volts, d'où la faible probabilité de déclenchement simultané de tous les groupes.

Le canal de fuite, précédé d'un court troncon en galerie, est à écoulement libre, creusé dans les alluvions de l'Arc sur la plus grande partic de son tracé.

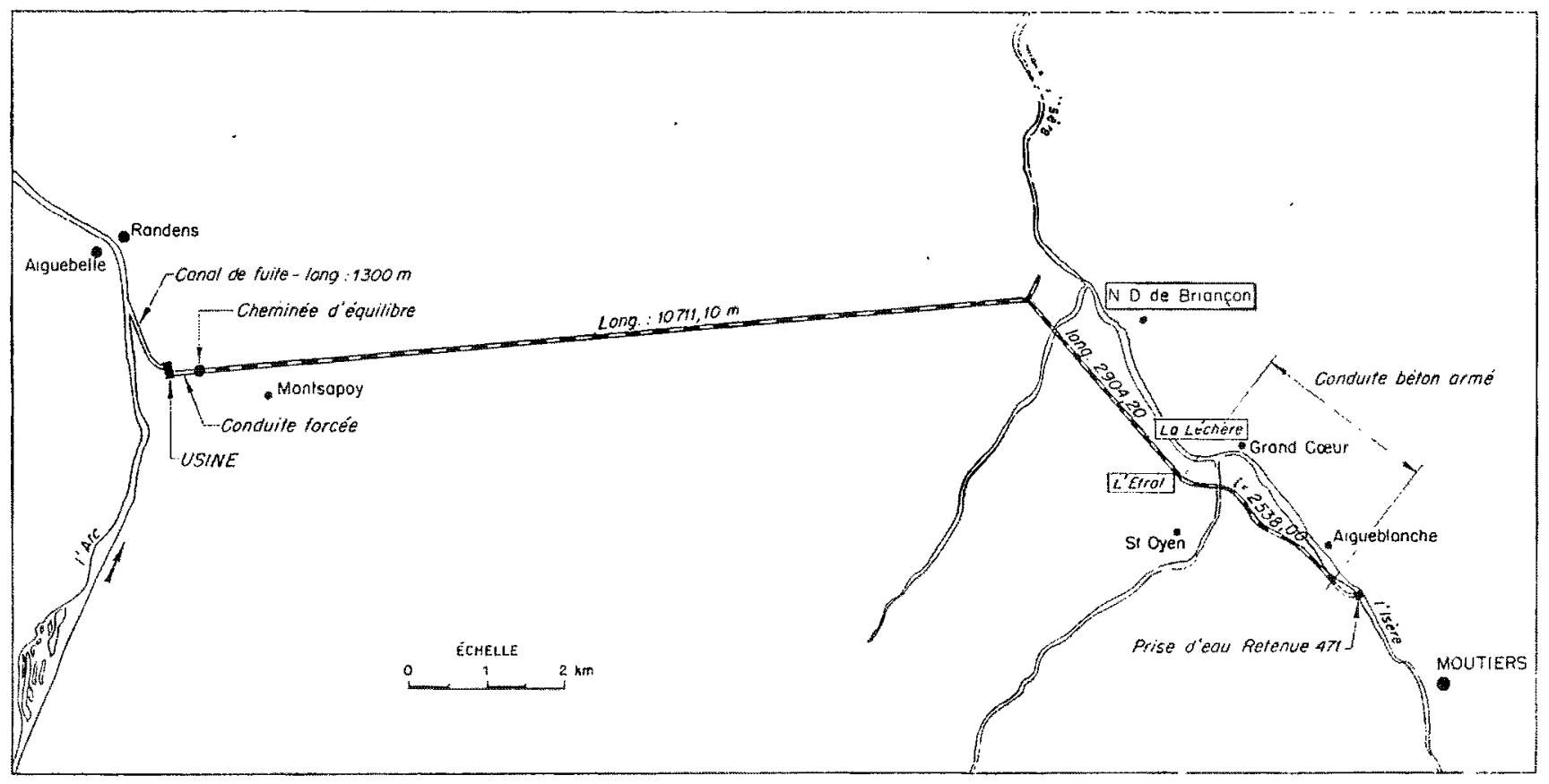

Fici, 1

Le tracé génóral des canalisations est reportẻ sur la figure 1.

L'énergie, emmagasinée du fait de la vitesse de l'eau dans l'ouvrage d'amenéc, atteint une valeur considérable et la cheminée d'équilibre correspondante était, de toutes façons, destinée à recevoir des dimensions importantes surtout si l'on voulait, eu égard à la hauteur de chute, limiter la montée de l'eau à une valeur acceptable pour en diminuer les répercussions sur le dimensionnement des canalisations et sur les conditions de régulation.

\section{Etude des pertes de charge}

On connaît le rôle que jouent les pertes de charge lors de l'oscillation en masse dans un système galerie-chemince d'éfuilibre; aussi, nous avons essayé d'approcher le plus possible to résultat probable en faisant procéder à un essai expérimental, dans la galerie d'amence de Cordéac, de caractéristiques assez voisines (diamétre $5,40 \mathrm{~m}$ ) et dont les conditions d'exécution sont sensiblement identiques (emploi systématique de coffrages métalliques). Le coefficient de 
rugosilé exprimé par la formule de STrickler a clé lrouvé ćgal à 75; malgré cette détermination expérimentale, nous avons gardé une fourchette assez importante entre les pertes de charge admises à la montée et à la descente, soit 14 et $20 \mathrm{~m}$, ce qui correspond, compte tenu des pertes singulières, à des coefficients de perte de charge (STRICKLER), compris entre 71 et 80 .

\section{II. - CHOIX DU TYPE DE CHEMINÉE}

La solution en chambres d'expansion, supérieure et inférieure, sans étranglement, ne fut pas examince en détail. On sait, en effet, que ce type d'ouvrage est intéressant quand le puits central peut être de dimensions réduites et quand les chambres d'expansion peuvent être calées haut el c̀tre très plates; ce n'était pas le cas à Randens, étant donné les dimensions qu'on aurait dù domner au puits central pour, au moins, ne pas être trop en dessous de la section de 'Thomas. D'autre part, des chambres d'expansion tròs plates auraient été d'une longueur démesurée, incompatible avec les conditions de remplissage rapide au cours d'une fermeture.

Le type différentiel pouvait, à première vuc, paraitre séduisant, étant donné la dissymétrie entre la valeur optimum de la perte de charge à créer par l'étranglement à la fermeture et à l'ouverture, beaucoup plus partielle. Il est, en effet, possible dans ce cas de dimensionner l'étrangle. ment du grand puits pour l'ouverture, la communicalion étant réalisće lors du déclenchement par le puits différentiel. Une solution séduisante pouvait mòme consister à prévoir le petit puits directement dans le prolongement des conduites forcées soulerraines. Le génie civil restait cependant assez compliqué du fail des difficultés provenant des robinets de lête de conduite. En ontre, le puits différentiel était assez sensible aux fausses manceuves, une ouverture trop importante pouvant notamment provoquer sa vidange rapide, done une grosse entréc d'air dans les conduites.

Restaient les solutions déversantes et à étronglement, pour lesquelles différents projets de chaque type furent établis. Ils servirent de base à des duludes comparatives assez poussées. Au point de vue prix, la comparaison était renduc assez alcatoire en raison de l'estimation incertaine concernant, d'une part, la répercussion d'une augmentation de charge sur le prix des ouvrages d'amence, d'autre part, le coût de l'ouvage de dissipation d'énergie, pour lequel it n'existait pas de précédent bien comparable. En définitive, nous avons conservé la solution à changlement, qui nous semblait plus sûre.

La perte de charge de l'étranglement n'était pas cncore strictement fixée; sa valeur maximum à la fermeture était donnée par l'étranglement oplimum créant à la fermeture une perte de charge maximum pour le plein débil égal à la montée maximum au-dessus du niveau statique augmenté des pertes de charge dans la galerie, soit $31 \mathrm{~m}$ environ.

Si l'on veut satisfaire sans variation de diamètre aux conditions d'ouverture, on est conduit à une section de $200 \mathrm{~m}^{2}$, correspondant à un diamètre de $16 \mathrm{~m}$, donc à une dimension do puits qui, tout en étant respectable, pouvait être exécutée sans difficulté spéciale. Nous avons, en effet, considéré comme exécutable un puits de diametres $15-17 \mathrm{~m}$, mais nous avons admis qu'au-delà il serait nécessaire de compléter la section du puits par des galeries horizontales donnant la surface désirée. Ce sont done les conditions relatives à l'ouverture qui ont, en définitive, donné la dimension minimum du puits central. D'ailleurs, il est possible que la centrale de Randens débite en hiver sur un réseau dont la puissance ne sera pas beaucoup supérieure au triple de sa puissance propre, et le fait que d'autres raisons nous conduisent a adopter une section double de celle de Thomas $\left(95 \mathrm{~m}^{2}\right)$ a l'avantage d'éviter toute discussion au sujet de la stabilité des petites oscillations.

Type d'étranglement. - Etant donné la dissymétrie entre les conditions d'ouverture el de fermeture, il est bon de donner à l'étranglement de la cheminée la dissymétrie maximum; d'autre part, pour la commodité des travaux, il est préférable de déjeter la cheminée par rapport à la galerie car la grande longueur du troncon. sans fenêtre nécessitera pendant toute la durée des travaux une circulation intense qu'il n'est pas possible d'interrompre en excavant un puits à Ia calotte de la galerie d'amenée. Pour augmenter au maximum les possibilités d'abaissement du niveau dans la cheminée, une disposition un peu spéciale de l'étranglement a été proposée; elle est schématiquement représentée sur la figure 2. Elle présente la particularité de mettre la galerie en dépression, uniquement en régime transitoire, ce qui ne semble pas présenter d'inconvénient; il parut indispensable d'essayer ce système d'étranglement sur modèle réduit pour en vérifier l'efficacité et la dissymétrie; cette étude fut réalisée au Laboratoire National d'Hydraulique à Chatou. Cependant, cet étranglement présente un certain inconvé- 


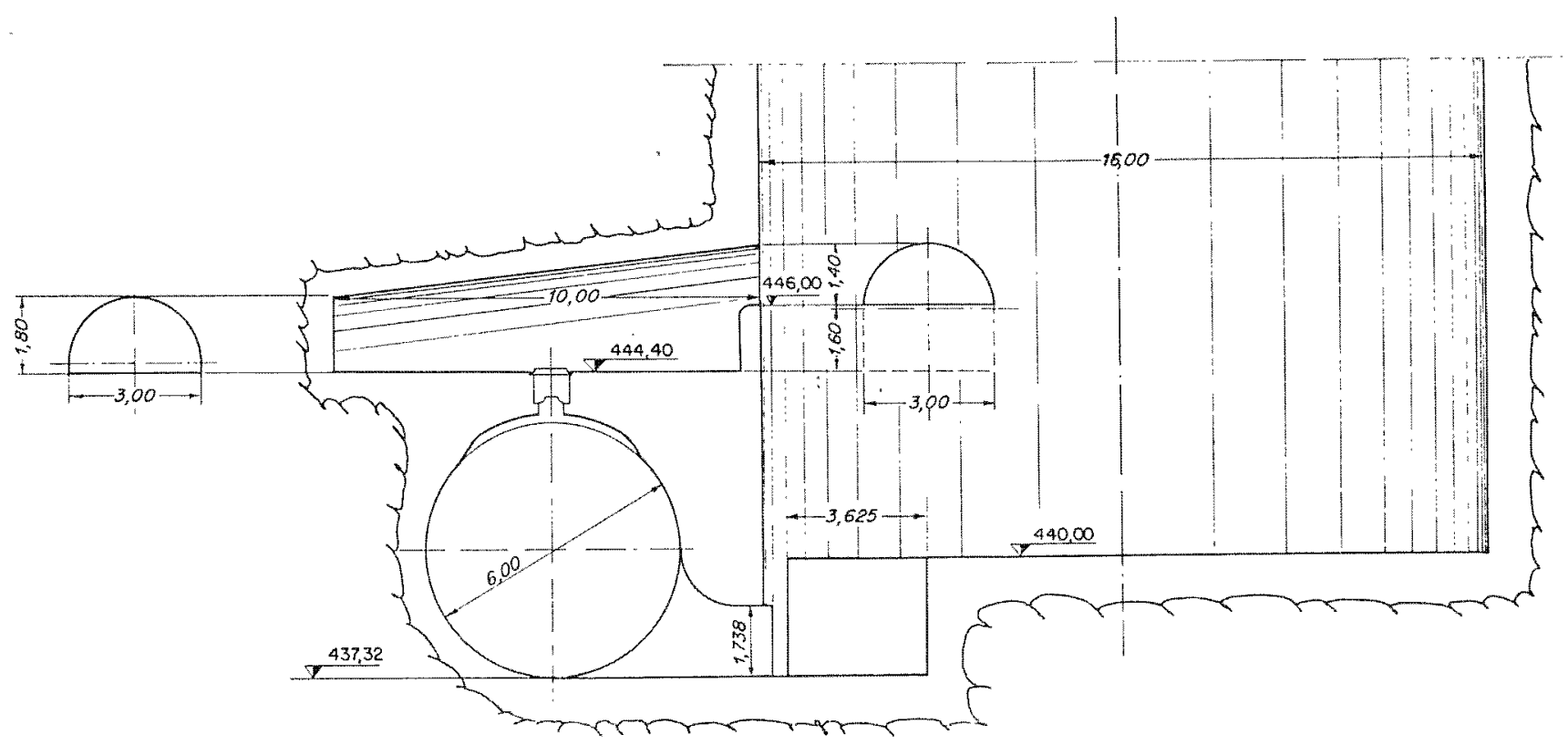

Fra, 2

nient vis-à-vis des bulles d'air entrainées par l'eau dans la galerie; ces bulles ne peuvent pas s'échapper au droit de la cheminée et risquent à la longue de former, à cet endroit-là (change- ment de pente) une bulle importante avec tous les inconvénients qui peuvent en résulter; e'est pourquoi il a été prévu un dispositif spécial constituant un désaérateur (fig. 2).

\section{III. - CALCUL DE LA CHEMINÉE A ÉTRANGLEMENT}

Comme on vient de le voir, il avait été décidé que la cheminée d'équilibre serait formée d'un puits central de 15 à $17 \mathrm{~m}$ de diamètre sur lequel serait branché un certain nombre de chambres d'expansion (fig. 3), de façon que la

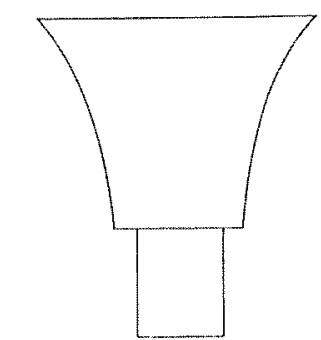

Forme theorrque donnee por le colcut

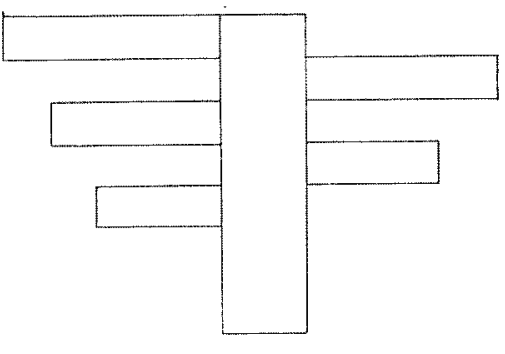

Fin. 3 chombres d'expanstons section résultante soit celle que donnerait le calcul (ainsi, un calcul grossier pour une cheminée à étranglement optimum donne une section de l'ordre de $450 \mathrm{~m}^{2}$ qui équivalait à un diamètre de $24 \mathrm{~m}$ ). D'autre part, cette disposition permet. sans difficulté, de construire une chemince d'équilibre dont la section varie d'une manière presque continue en fonction de la cote, ce qui permet, comme nous le verrons plus loin, de conserver une valeur constante à la pression au droil de la cheminée d'équilibre, au cours d'une mancuvre de fermeture.

La forme de la cheminée étant dégrossie, le calcul a été conduit de la manière suivante :

Le diamètre du puits a élé calculé pour satisfaire aux conditions d'ouverture, en admettant que la partie basse de la cheminée d'équilibre (celle qui est le plus souvent en pression) serait constituce de la manière la plus simple par un puits cylindrique sans chambre d'expansion. Les chambres d'expansion de la partre supćrieure ont été calculées par la méthode directe relative à la condition de fermeture totale instantance et permettent ainsi de serrer, le plus près possible, la forme idéale qui n'est, en général, pas celle d'un cylindre. Notons en passant que la partie basse de la cheminée d'équilibre n'est pas définie avec la même exactitude que la partie haute puisque les conditions d'ouverture sont beaucoup moins bien définies que les conditions de fermeture. 


\section{$1^{\circ}$ Calcul à l'ouverture - Diamètre du puits}

Si les conditions de fermeture sont bien définies, les conditions d'ouverture ont fait l'objet d'une étude spéciale; il ne ponvait être question d'admettre une ouverture complète instantanée, qui aurait conduit soit à surdimensionner considérablement la base de la cheminée d'équilibre, soit à caler la galerie à une cote trop basse (cc qui aurait eu pour conséquence de trop la surcharger). La situation de cet aménagement n'est pas telle qu'il soit justifiable d'une pareille mesure. La réserve est de faible importance et l'usine doit surtout pouvoir turbiner les lâchures d'hiver du barrage de Tignes, dont l'usine principale Malgovert est équipée à $50 \mathrm{~m}^{3} / \mathrm{s}$.

En définitive, la cheminée d'équilibre doit permettre la mise en marche rapide de chacun des quatre groupes et, en particulier, la mise en marche du quatrième et dernier groupe. Cette condition étant remplie, il est bien évident que la cheminée d'équilibre permettra, à plus forte raison, l'ouverture des trois premiers (séparćment). Nous avons également calculé la chemince d'équilibre de manière qu'elle permette la mise en marche des quatre groupes à la pleine charges en $8 \mathrm{mn}$, ce qui semble conduire à des normes d'exploitation correctes. Or, au momenl où nous faisions cette étude, le modèle réduit de l'étranglement était encore à l'étude et nous ne connaissions pas exactement ses caractéristiques.

Cependant, il faut remarquer qu'une ouverture de 75 à $100 \%$ n'intéresse que le quart du débit total, donc que l'étranglement crée une dépression maximum qui n'est que le $1 / 16$ de la dépression qu’il créerait pour le débit total. Autrement dit, pour cette manœuvre particulic̀re, son influence est faible et pratiquement négligeable. Il en est de mème d'ailleurs pour l'ouverture progressive des quatre groupes en $8 \mathrm{~mm}$ qui ne fait intervenir en travers de l'étranglement que des débits inférieurs à $25 \mathrm{~m}^{3} / \mathrm{s}$. Donc, pratiquement, il n'était pas indispensable de connaître exactement les possibilités de l'étranglement.

En définitive, nous avons pris un puits de $\varnothing 16 \mathrm{~m}$ permettant l'ouverture de 75 à $100 \%$ et l'ouverture progressive en $8 \mathrm{mn}$ dans le cas le plus défavorable où l'étranglement n'aurait donné que $30 \mathrm{~m}$ de perte de charge pour $\mathrm{Q}=100 \mathrm{~m} 3 / \mathrm{s}$ (dissymétrie de l'étranglement ćgale à 2, ce qui pouvait déjà être considéré comme un minimum). Ces conditions auraient donc été, à plus forte raison, remplies si l'étranglement avait donné une perte de charge supérieure à $30 \mathrm{~m}$, comme les résultats partiels de l'essai sur modèle nous le laissaient espérer.
La puissance de l'étranglement conserve cependant un énorme intérêt pour les manouvres d'ouverture de 0 à $x \%$ qui sont particulièrement intéressantes. En effet, après un déclenchement général, il est souvent bon de pouvoir brancher, le plus vite possible, une certaine fraction de la puissance de l'usine sur le réseau, le reste étant donné plus progressivement quelques instants après. Cette valeur de 0 à $x \%$ n'est pas définie avec autant de précision que la condition d'ouverture de 75 à $100 \%$, mais il est ćvident que, toutes choses égales par ailleurs, il $\mathrm{y}$ a intérêt à chercher la dissymétrie maxi-

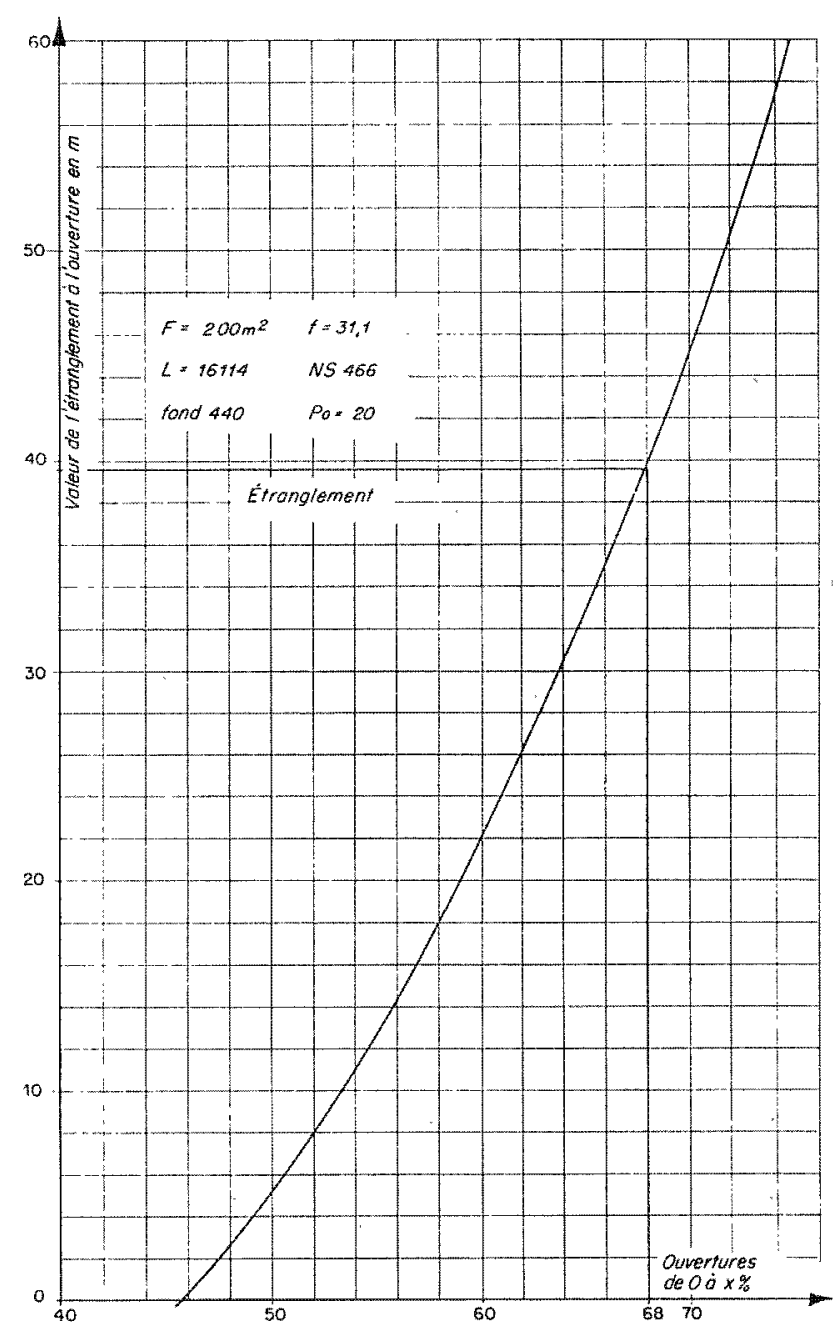

FIG. 4

mum, c'est-a-dire, pour une caractéristique donnée de l'étranglement à la fermeture, la déprespression maximum à l'ouverture. Le graphique joint, fait à l'aide des abaques Calame et GADFN, montre, pour $\varnothing 16 \mathrm{~m}$, les possibilités d'ouverture de 0 à $x \%$ en fonction de la valeur de l'ćtranglement $R_{0}^{\prime}$ à l'ouverture (fig. 4). 


\section{$2^{\circ}$ Calcul à la fermeture complète par le procédé direct et détermination de l'étranglement}

Le diamètre du puits étant fixé, nous nous sommes également donné la cote maximum d'eau dans la cheminée d'équilibre, soit 488. Pour un étranglement de valeur $R_{0}$ donné, le procédé direct permet de trouver la forme idéale des cham- bres d'expansion de telle manière qu'au cours d'un déclenchement, à partir du moment où l'eau atteint le radier de la chambre d'expansion la plus basse, la pression sous l'étranglement reste constante et égale à la montée maximum de l'eau dans la cheminée d'équilibre (fig. 5).

Comme nous l'avons expliqué dans l'article du $\mathrm{n}^{\circ} 3 / 1951$ de la Houille Blanche, une telle cheminée d'équilibre a un volume à peine supé-

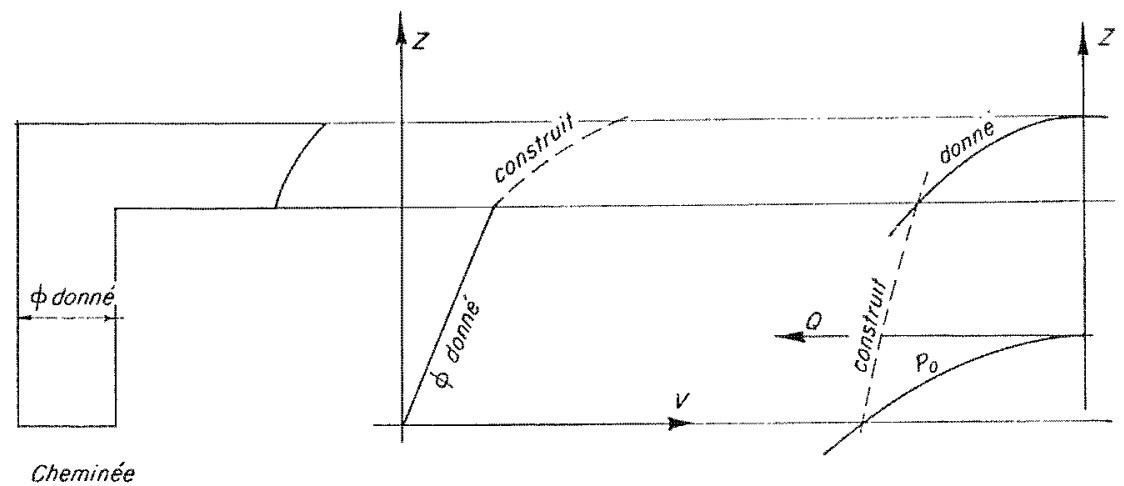

Fy. 5

rieur à la cheminée d'équilibre à étranglement. optimum (elle pourrait même avoir un volume inférieur avec un diamètre de puits plus petit); mais elle a sur cette dernière le gros avantage d'avoir un étranglement créant une perte de charge moins forte. Or, dans le cas de Randens, la perte de charge maximum étant de $14 \mathrm{~m}$ dans la galerie, la montée maximum de l'eau, au-dessus du niveau statique, étant de $17 \mathrm{~m}$, un étranglement optimum aurait créé une perte de charge de $17+14=31 \mathrm{~m}$ pour $\mathrm{Q}=100 \mathrm{~m}^{3} / \mathrm{s}$. Cette surpression, en cas de dé-

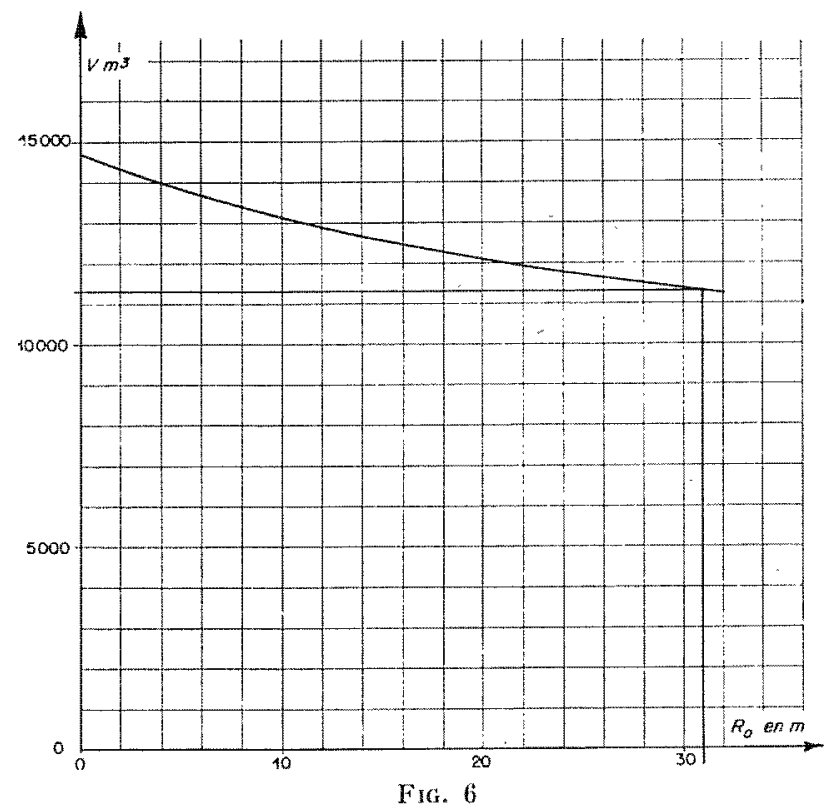

clenchement, aurait été appliquée en quelques secondes sur la galerie ( 4 s environ). Sans avoir de critère précis indiquant qu'à partir de telle ou telle surpression on risque de détériorer la galerie, il nous a semblé préférable d'adopter un étranglement moins puissant, d'autant plus que le volume de la cheminée d'équilibre étail à peine affecté par cette diminution.

Pour fixer notre choix, nous avons fait un certain nombre d'épures par la méthode directe qui nous ont permis de tracer la courbe de la figure 6, qui donne, pour chaque valeur de l'étranglement, le volume de la cheminée d'équilibre correspondante.

On voit qu'à une valeur de $R_{0}$ donnée correspond une cheminée d'équilibre de forme bien définie, donc des chambres d'expansion bien définies également. II nous a semblé qu'un seul étage de chambres d'expansion serait préférable à plusieurs; en eflet, l'aération d'une chambre d'expansion en charge est toujours délicate et nécessite des pentes importantes en radier et en calotte, gênantes pour la construction. D'autre part, un étage de chambres d'expansion en charge demande, à son intersection avec le puits, un ferraillage assez important et une forme, donc des coffrages assez compliqués. Compte tenu de la qualité du rocher, nous avons estimé qu'une chambre d'expansion de 8 à $9 \mathrm{~m}$ de haut pouvait être creusée sans revêtement; ceci nous conduisait done à un étranglement $R_{0}$ de $15 \mathrm{~m}$ et c'est, en définitive, à cette valeur moyenne, qui représente moins de la moitié de l'étranglement optimum, que nous nous som- 
mes arêtés. I'épure permet de construire la courbe des volumes qui n'est évidemment pas une droite $\left(^{\star}\right)$. Pour nous rapprocher pratiquement de cette courbe, nous y avons inscrit 2 segments de droites qui nous ont donné 2 sections qu'il a été facile de réaliser pratiquement à l'aide de 2 chambres d'expansion de hauteurs différentes. Nous donnons, figure 7 , le plan d'ensemble de la cheminée d'équilbre.

Une épure, dans le sens habituel (indirect), faite à partir de la courbe des volumes ainsi définie, nous a montré que la surpression à $20 \mathrm{~cm}$ près était constante et équivalente à un plan de charge à la cote 488 , tout au long de la montée de l'eau dans les chambres d'expansion au cours d'un déclenchement à puissance maximum.

Le volume total de cette cheminée est de $12.500 \mathrm{~m}^{3}$; le volume d'une cheminée cylindrique à étranglement optimum est de $12.200 \mathrm{~m}^{3}$, perte $3 \%$, alors que la valeur de l'étranglement est passée de $31 \mathrm{~m}$ à $15 \mathrm{~m}$ pour $\mathrm{Q}=100 \mathrm{~m}^{3} / \mathrm{s}$; d'autre part, la forme est plus simple, done moins onéreuse.

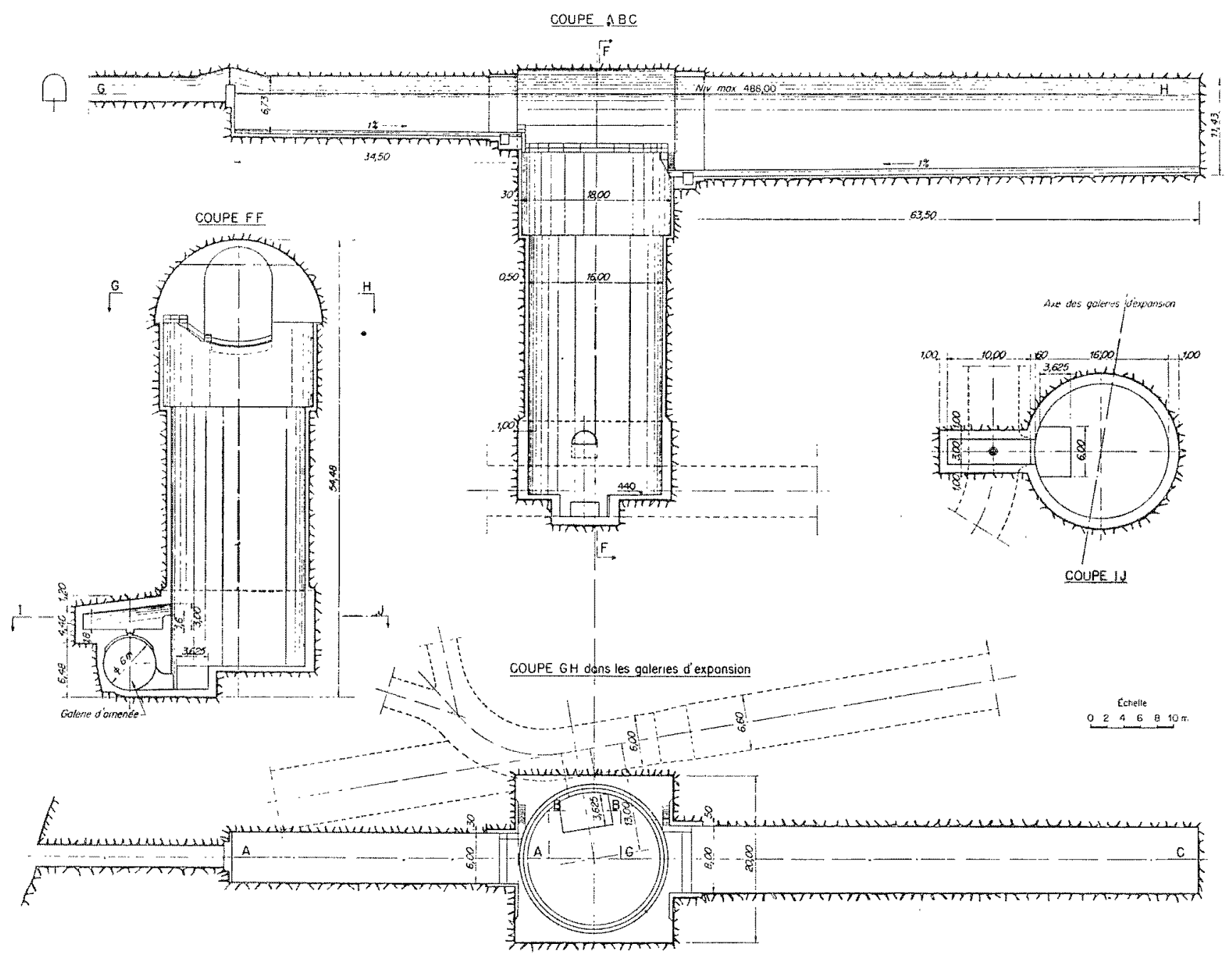

Fig. 7

\section{IV. - COUP DE BELIER CREE PAR L'ÉTRANGLEMENT DE LA GALERIE D'AMENEE}

Ainsi que l'a montré $Y$. Ponsar dans un article du $\mathrm{n}^{\circ} 2 / 1950$ de la Houille Blanche, une cheminée d'équilibre à étranglement risque, en cas de déclenchement brutal, lorsque la galerie

\footnotetext{
* Cetle épure a éte présentée dans le n० $3 / 1951$ de la Houlle Blanche; nous n'avons pas juge utile de la reproduire.
}

d'amenée est de grande longueur, de provoquer une onde de surpression qui remonte en vraie grandeur le long de la galerie jusqu’à une distance $a \mathbf{T} / 2$ de la prise d'eau :

$$
a=\text { Vitesse des ondes dans la galerie }
$$$$
\mathrm{T}=\mathrm{Temps} \text { de fermeture des turbines }
$$ 
c'est le cas de notre installation; les turbines peuvent en cas de déclenchement se fermer à la vitesse maximum des régulateurs, c'est-à-dire en 4 secondes, et la galerie a plus de $16.000 \mathrm{~m}$ de longueur. Une onde va donc remonter le long de la galerie jusqu'à une distance d'environ :

$$
\frac{a \mathrm{~T}}{2}=\frac{1.000 \times 4}{2}=2.000 \mathrm{~m}
$$

de la prise d'eau. Ceci est particulièrement grave car les $15 \mathrm{~m}$ de surpression se feront sentir jusqu'à la conduite en béton armé qui se trouve avant l'Etrat. Une épure de BERGERon confirma ces prévisions et montra méme que la surpression réelle était un peu plus forte que celle prévue, car, à la surpression produite par les $15 \mathrm{~m}$ de $R_{0}$, il fallait ajouter la montée de l'eau pendant les quatre premières secondes qui était d'environ $2 \mathrm{~m}$, ce qui portait à $17 \mathrm{~m}$ l'onde de surpression qui remontait le long de la galerie.

Remèdes envisagés. - Etant donné l'énorme augmentation du coût de la conduite en béton armé par mètre supplémentaire de pression d'eau à supporter ( 20 millions $/ \mathrm{m}$ ), il était indispensable de la protéger contre ces surpressions. Une première solution consistait à diminuer la force de l'étranglement. Cette solution a été vite abandonnée parce que :
- En dessous de $\mathrm{R}_{0}=10 \mathrm{~m}$, la forme optimum de la cheminée d'équilibre n'est plus du tout constructive, les chambres d'expansion deviennent trop basses et trop longues;

- Plus $R_{0}$ diminue, plus la montée de l'eau durant les quatre premières secondes est grande et il reste, de toute façon, un coup de bélier non négligeable dans la galerie d'amenée;

- Si $R_{0}$ est trop faible, les conditions d'ouverture de 0 à $x \%$, comme nous l'avons vu plus haut, deviennent très mauvaises et l'amortissement des oscillations très lent.

Une deuxième solution proposée par Neyrpic consistait à placer, à l'aval de la conduite, des batteries de soupapes antibélier; ces soupapes n'ayant jamais servi à une échelle aussi importante, cette solution n'a pas été retenue.

Enfin, la troisième solution que nous avons retenue - parce qu'elle est la plus simple et la plus sûre - a été de protéger la conduite en béton armé par un puits antibélier. La position et les dimensions de ce puits firent l'objel d'une étude spéciale dont nous allons maintenant retracer les principaux éléments.

\section{V. - ETUDE DU PUITS ANTI-BELLIER}

L'aération du puits antibélier conduisait à le placer a une profondeur pas trop faible afin de ne pas réaliser une fenêtre d'évacuation d'air trop importante. Cette conclusion était encore renforcée par les conditions d'exécution des travaux qui conduisaient à le placer, de préférence, au voisinage d'une attaque afin de ne pas trop perturber la marche des chantiers. Il était, en effet, possible de l'attaquer au moyen d'une fenêtre indépendante.

Pratiquement, on pouvait envisager trois posilions :

- A l'Etrat, à l'origine de l'attaque du tronçon de tunnel situé dans le lias, done à l'aval immédiat de la conduite en béton armé;

- Dans le cristallin, au voisinage immédiat de la Léchère, où l'attaque aura une couverture minimum;

- Enfin, à Notre-Dame-de-Briançon, où se trouve une fenêtre d'attaque qui aurait pu être utilisée pour l'aération.

La position du puits antibélier, ses dimensions, sa forme, étaient autant d'inconnues qu'il 
nous a fallu lever et qui nous ont conduits à des études assez longues et variées que nous allons exposer succinctement.

Ce puits antibélier participera lui-même à l'oseillation en masse dans le systeme galeriecheminée, d'où la nécessité de l'éludier à ce point de vue d'abord. Une lois ses dimensions déterminées, des épures de BERckion nous permeltront d'étudier la marche des ondes de pression.

Le processus d'étude est en somme le même que pour une cheminée d'équilibre, qui sert principalement à diminuer l'intensité des coups de bélier d'onde, mais qui est calculée surtout du point de vue de l'oscillation en masse.

\section{Etude théorique \\ en supposant les pertes de charge négligeables (oscillation en masse)}

11 est certain que l'hypothèse consistant à négliger les pertes de charge ne peut conduire à des résultats quantitatifs puisqu'elles jouent un ròle considérable dans les phénomènes d'oscillation en masse. Les résultats de cette étude, bien que qualitatils, nous ont cependant conduils à des conclusions intéressantes, que des ćludes parallèles, mais d'une portée moins générale, faites en tenant compte des pertes de charge, ont d'ailleurs entièrement confirmées.

On sail que l'oscillation dans les cheminées est représentée par les équations suivantes :

$$
\begin{aligned}
& Z_{1}=A \sin \alpha t+B \sin \beta t \\
& Z_{2}=A^{\prime} \sin \alpha t+B^{\prime} \sin \beta t
\end{aligned}
$$

$Z_{1}$ représente lordonnée du niveau dans la cheminée et $Z_{2}$ l'ordonnée du niveau dans le puits intermédiaire (en reprenant les notations indiquées par M. Escande dans son ouvrage sur les cheminées d'équilibre).

$\mathrm{AA}^{\prime}, \mathrm{BB}^{\prime}, \alpha$ el $\beta$ sont calculés à partir de formules relativement simples; l'oscillation est représentée par la superposition des mouvements, l'un de longue période correspondant à la pulsation $\alpha$, l'autre de courle période correspondant à la pulsation $\beta$.

Il n'est, en toute rigueur, pas évident que le niveau maximum atteint lors de l'oscillation en masse soit égal, pour chacun des ouvrages, à la somme des valeurs absolues des amplitudes des deux sinusoïdes de pulsations $\alpha$ et $\beta$, spécialement dans le cas où $\alpha$ el $\beta$ sont commensurables.

Nous n'avons pas cherché a examiner en détail cette question qui nous faisait entrer dans des considérations dépassant le cadre du calcul des cheminées d'équilibre. Dans le cas qui nous occupe, $\alpha$ et $\beta$ sont d'un ordre de grandeur tel que ( $\alpha$ chant de l'ordre de grandeur de $10 \beta$ ), les montées maxima sont pratiquement représenlées par $|A|+|B|$ et $\left|A^{\prime}\right|+\left|B^{\prime}\right|$.

a) INFLUENCE DE LA POSITION DU PUITS ANTIBÉLIER. - Nous avons supposé que l'emplacement et les dimensions de la cheminée aval élaient bien déterminés el nous avons examiné quelle était l'influence de la position du puits antibélier prise comme variable. Si le niveau dans le puits antibélier se bornait à suivre la pression dans la galerie, l'ordonnée maximum atteinte, lors de la montée du niveau d'eau dans la cheminée d'équilibre aval, serait égale à $\mathbf{K Z}$, K représentant le rapport de la distance du puits antibélier à la prise d'eau sur la longueur totale de la galerie, $Z$ la montée maximum dans la cheminée.

La diffcrence $\left(A^{\prime}+B^{\prime}\right)-K Z$ représente, dis

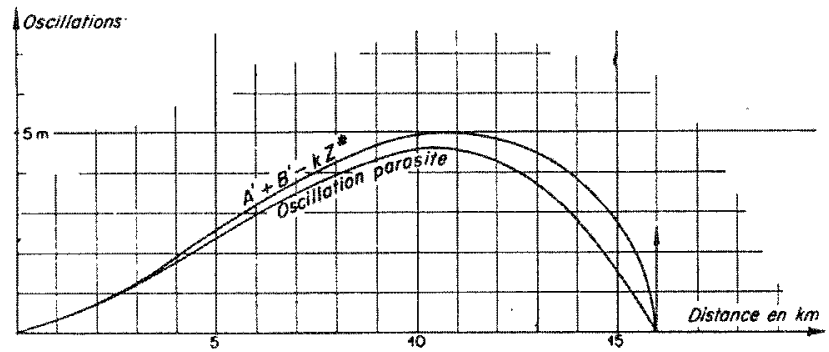

Fra. 8

lors, ce que nous avons appelé « l'oscillation parasite » dans le puils antibélier, c'est-àdire le supplément de montée dù à l'oscillation en masse. Nous avons tracé cetle variable dans la courbe représentée sur la figure 8 . Cette courbe a une allure pouvant être prévue $a$ priori; si le puits antibélier est au voisinage immédiat de la prise d'eau, l'oseillation parasite est nulle puisque, le niveau amont étant supposé fixe, le niveau du puits antibélier ne peut guère osciller, par suite de la rapidité des accélérations dans le troncon intermédiaire de galerie. Si, au contraire, le puits antibélier est situé près de la cheminée d'équilibre, il se bornera ćgalement a en suivre les oscillations pour la même raison.

En définitive, l'oscillation parasite conduirait à des surpressions dans la zone amont sensiblement identiques pour les trois positions.

b) INFLUENCE DE LA SEction DU PUITS ANTIBÉlier. - Nous avons étudié, suivant les mêmes bases, quelle était l'influence de la section du puits antibélier sur le mouvement; on aurait pu, en eflet, supposer, à première vue, qu'un accroissement de la section pouvait diminuer l'amplitude de la montée dans la cheminée d'équilibre. Nous avons supposé, dans cette étude, pour simplifier, que le puits antibélier était au quart de la distance entre la prise d'eau et la cheminéc 
d'équilibre. Le calcul nous a conduits aux tésullats représentés sur la courbe de la figure 9.

Celte courbe montre immédiatement que l'accroissement de section du puits antibélier ne joue pratiquement pas de rôle sur la montée dans la cheminée, mais que, par contre, la montée dans le puits antibélier est considérablement renforcée.

La demi-oscillation ne part pas de l'origine car, de toutes facons, l'amplitude dans le puits est au moins égale au quart de l'amplitude dans la cheminée d'équilibre, si sa section tend vers 0 (tubes piézométriques). Nous avons effectué les calculs pour un rapport $F_{1} / F_{2}$ supérieur à 1 , bien que ce cas ne soit pas intéressant dans la pratique. Si $F_{2}$ voit sa section croitre indéfiniment, il est certain que l'oscillation tend vers 0 , puisque cela revient à déplacer le niveau statique au droit du puits. La courbe passe donc nécessairement par un maximum; il résulte done de cette étude qu'il faut donner au puits antihélier la section minimum compatible avec le rôle qu'il a à jouer vis-à-vis des coups de bélier. Nous avons admis que sa section devrait être de l'ordre de grandeur de celle de la galerie d'amenée et nous nous sommes arrêtés à un ouvrage circulaire de $6 \mathrm{~m}$ de diamètre.

c) ETUde Do type D'ouvrage. Ouvrage déversant.

Pour que le déversoir soit intéressant, il faul évidemment qu'il soit arasé à une cole notablement inférieure au niveau maximum alleint lors de l'oscillation en masse correspondanl au diamètre du puils que nous avons choisi, ce qui revient pratiquement à l'araser au niveau slatique ou très peu au-dessus. Si nous considérons la vitesse de l'eau dans la galerie entre la jrise d'eau et le puits déversant, elle sera extrêmement peu freinée par la montée de l'eau dans louvrage. La hauteur accélératrice sera représentée uniquement par la montée du niveau audessus du déversoir; elle sera done très faible. Le débit ne décroitra donc seulement que dans le troncon amont de la galerie. Par contre, lo freinage dans la portion de la galerie comprise entre le puits antibélier et la cheminée sera accru du fait que la longueur de la galerie sera plus réduile.

Quand le niveau dans la cheminée d'cquilibre descendra lors d'une fermeture complète, le débit du déversoir sera égal au déhit dirigé dans le sens amont aval du troncon amont de galerie qui n'aura pas eu le temps d'être freiné, augmenté du débit dans le sens aval amont dans le troncon aval de galerie. Théoriquement, en supposant le déversoir arasé rigoureusement au niveau statique et le débit du déversoir égal à $100 \mathrm{~m}^{3} / \mathrm{s}$ pour une surélévation très faible, on déverserail ainsi $200 \mathrm{~m}^{3} / \mathrm{s}$. Dans le cas qui nous intéresse, le débit déversé aurait ćté un peu plus réduit, mais il atteignait encore une valcur inadmissible qui anrait conduit à prévoir des ouvrages de dérivation très importants, sans compler des pertes d'eau considérables; c'est pourquoi nous avons abandonné celle solution.

\section{Oubrage i deax sections.}

Nous avons vu que si le puits a une grande section sur foute sa hateur, la montée est amg.

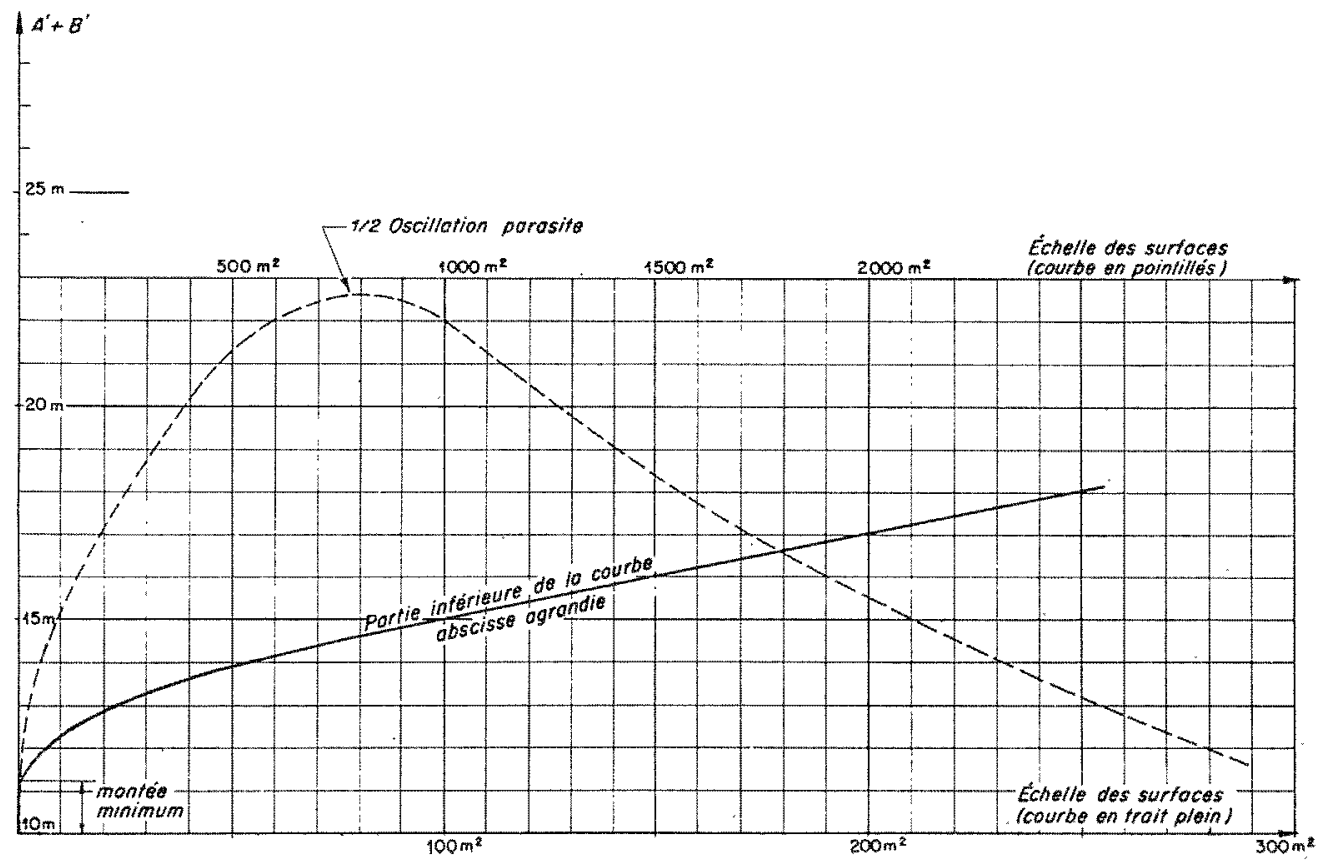

FIG, 9 

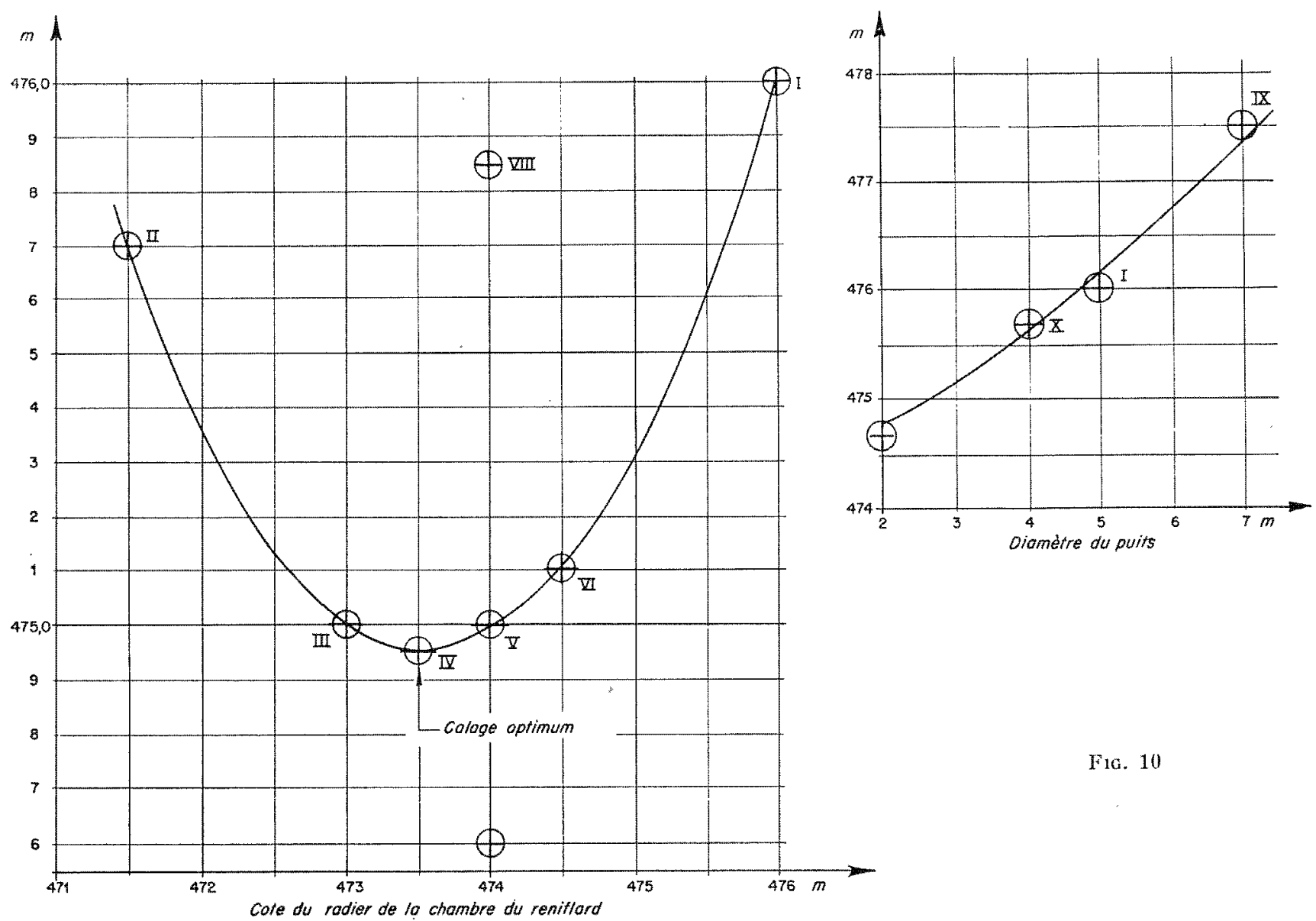

mentée. Si, par contre, on lui donne une section agrandie au voisinage immédiat de la montée maximum correspondant au puits de $\varnothing 6 \mathrm{~m}$, on aura tendance à diminuer la surélévation. Il doit donc exister, pour une section de petit puits donnée et pour une section de chambre d'expansion supérieure donnée, une position optimum telle que la montée résultante se trouve diminuée au maximum. C'est ce que nous avons vérifié quand l'emplacement et le diamètre du puits ont été fixés.

On était donc conduit aux résultats suivants :

1. La protection créée par le puits antibélier sur la zone amont est pratiquement indépendante de sa position le long de la galerie d'amenée.

$2^{\circ}$ Les ouvrages déversants ou à étranglement ne sont pas intéressants;

$3^{\circ}$ Il faut done donner au puits une section minimum compatible avec l'amortissement du coup de bélier;

$4^{\circ}$ Il est intéressant de réaliser un ouvrage à deux sections en calant le changement de section à une cote optimum à calculer, compte tenu des pertes de charge.

\section{$2^{\circ}$ Etude d'oscillation en masse en tenant compte des pertes de charge}

Ces conclusions découlaient cependant d'une étude faite en négligeant les pertes de charge. Etant donné le rôle fondamental qu'elles jouent dans les phénomènes d'oscillation en masse, il était indispensable de les confirmer au moyen d'épure tenant compte de l'ensemble du phénomène.

Ces épures confirment bien, dans leur ensemble, les conclusions qualitatives que nous avions obtenues précédemment; pratiquement, la surpression dans la canalisation comprise entre la prise d'eau et le puits est sensiblement indépendante de la position de ce dernier. Puisque la section du canal est constante, cette surpression est obtenue simplement en reportant la surpression au puits proportionnellement aux distances puisqu'il n'y a plus, dès lors, de phénomènes de coups de bélier.

Ftant donné que la hauteur du puits antibélier croit à mesure qu'on le déplace vers l'aval, puisque l'oscillation est un peu plus grande d'une part, et que, d'autre part, le radier de la galerie s'abaisse, il est intéressant de le mettre 
le plus en amont possible; il ne semblait donc pas nécessaire de le placer ailleurs qu'à l'Etrat, ce qui conduisait évidemment au coût minimum, puisque l'ouvrage avait la hauteur minimum du fait de sa proximité de la prise d'eau.

La position et le diamctre du puits antibélier ctant fixés, nous avons fait un certain nombre d'épures pour déterminer le calage optimum de la chambre d'expansion. Le résultat de ces épures a été traduit sur les courbes ci-jointes (fig. 10) (*). La forme définitive du puits antibélier est représentée sur la figure 11.

* Cette courbe et les épures correspondantes ont été faites par le bureau Etudes et Entreprises d'Aix-lesBains.

\section{3" Etude du système par la méthode Bergeron}

La raison d'être du puits est l'extinction des coups de bélier en amont, l'oscillation en masse ćtant parasitaire. Aussi était-il indispensable de l'ćtudier en détail.

Nous avons done ellectuć quelques épures de Bergrron sur le système complet galeriecheminée d'équilibre-puits intermédiaire, alin do vérifier si le fait de négliger la propagation de la perte de charge de l'étranglement par coups de bélier n'entachait pas d'erreurs systématiques les calculs d'oscillation en masse. L'épure que nous donnons ci-joint montre que l'ordre de grandeur du phénomène est respecté (épure 1).

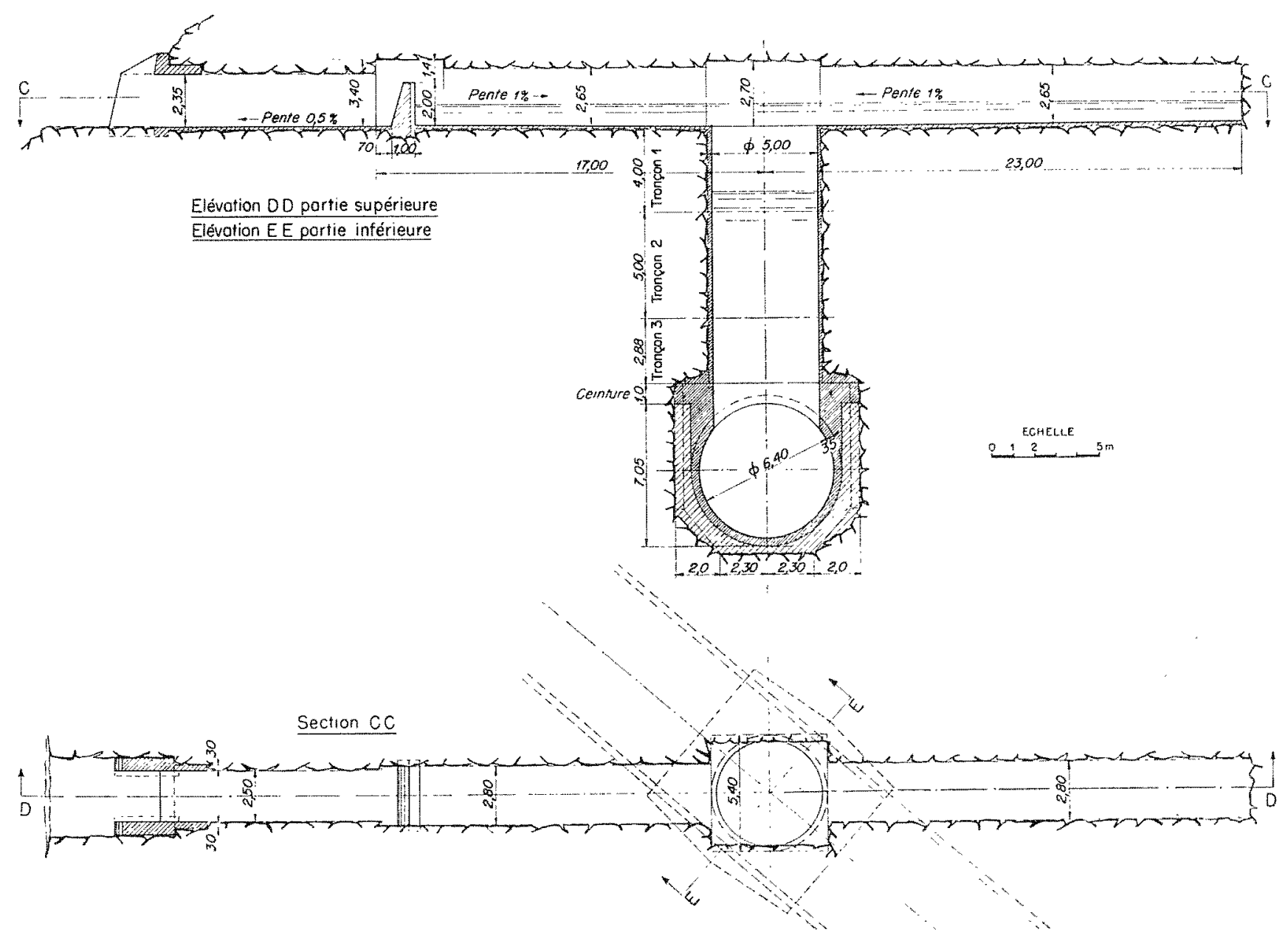

Fig. 11. Disposition du puits anti-bélicr. 
- - - Epure d'oschlotion en masse

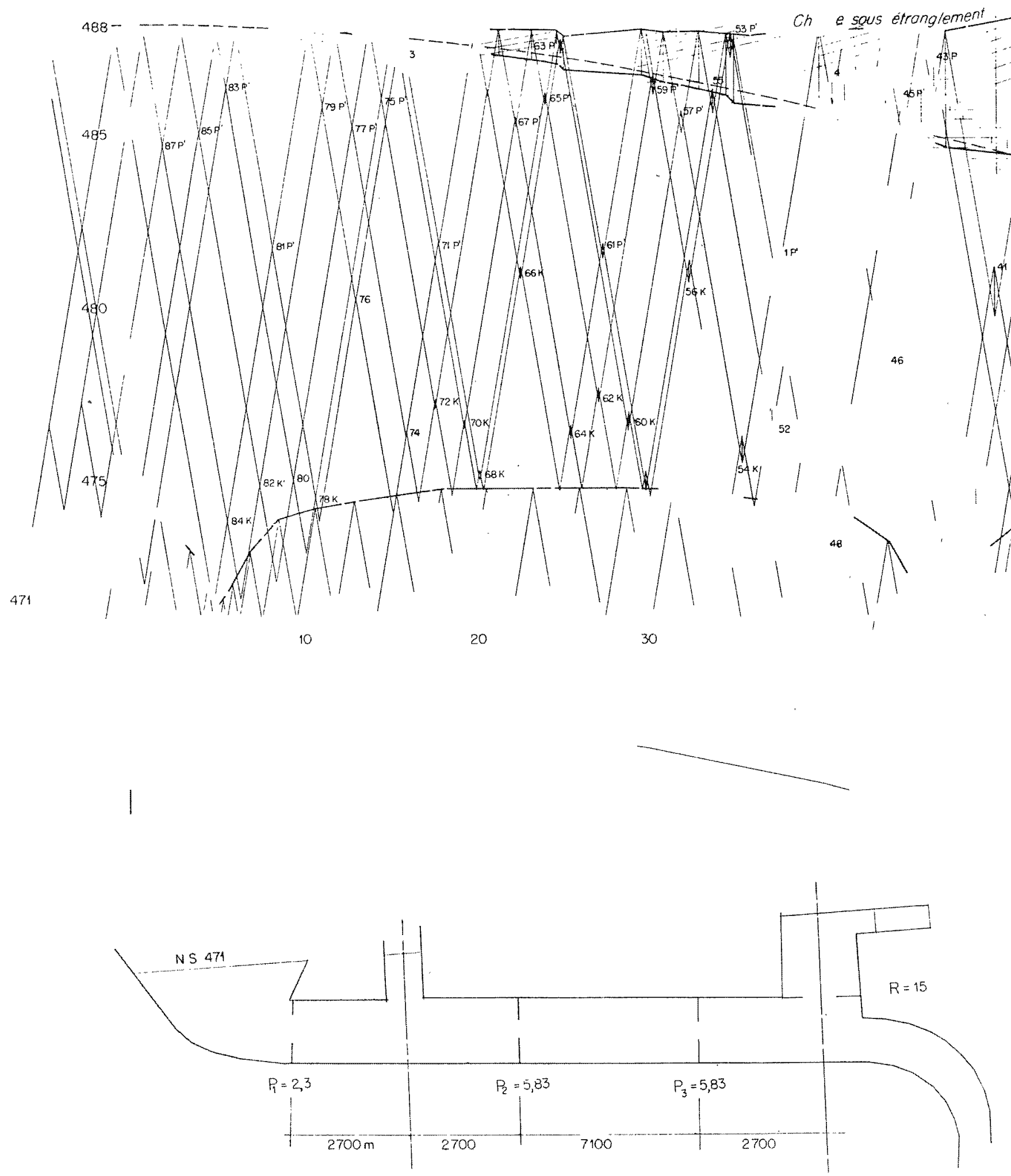




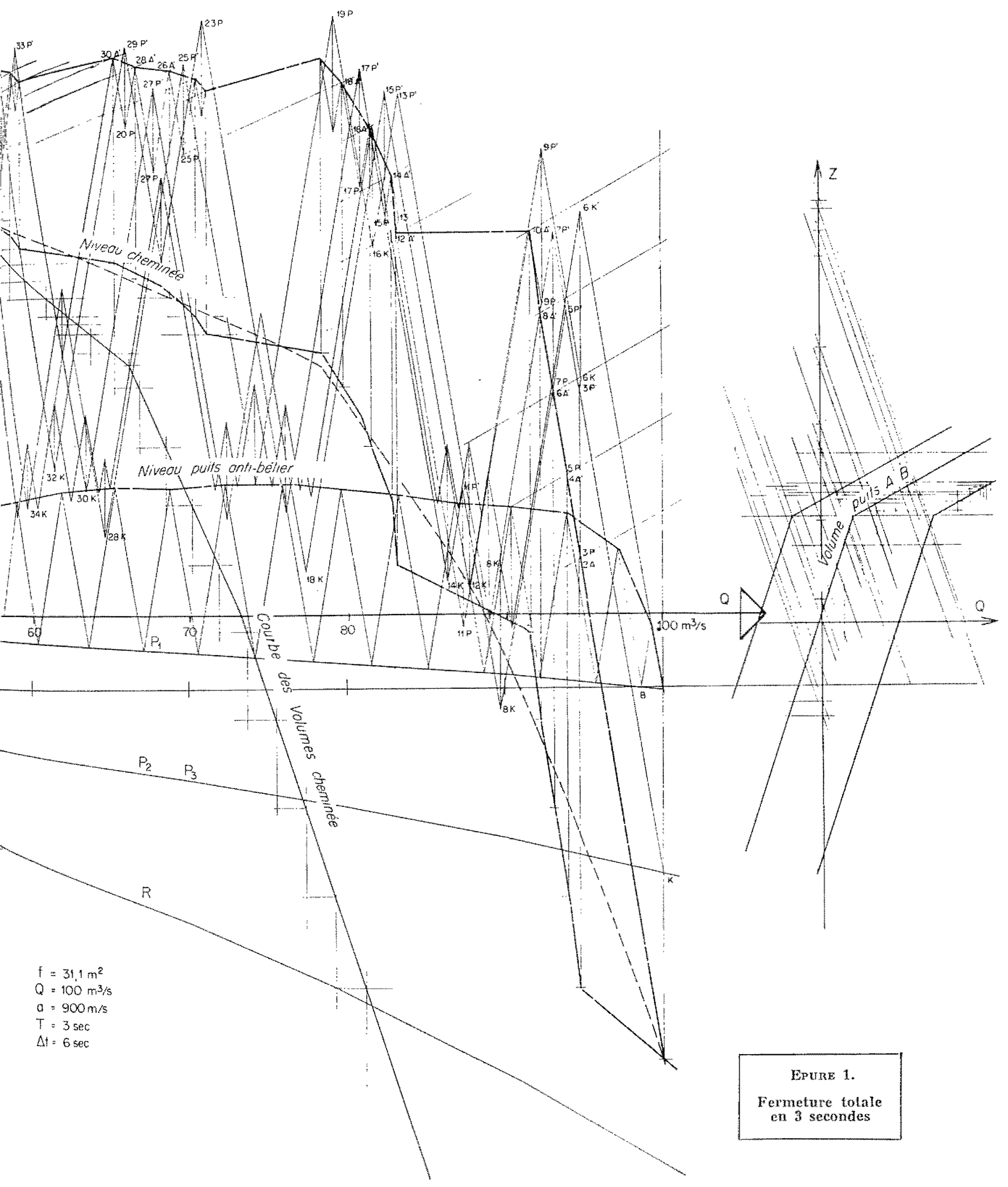




\section{VI. - ETUDES PARTICULIERES}

Les dimensions principales de la cheminéc d'équilibre et du puits antibélier ainsi définies, nous avons procédé à des vérifications complémentaires destinées à préciser le fonctionnement des ouvrages dans certaines hypothèses de fonctionnement.

Nous exposerons d'abord brièvement les résultats de l'essai sur modèle réduit réalisé à Chatou.

L'objet essentiel de cel essai était de déterminer un étranglement créant $15 \mathrm{~m}$ de perte de charge à la montée et la perte de charge maximum à la descente de l'eau; de plus, cet étranglement devait être de forme simple et facile à réaliser pratiquement.

La forme retenue est représentée sur la figure 2. Les performances de ce dispositif, Iransmises par le Laboratoire, sont indiquées dans Ie tableau ci-contre:

On remarque que le coefficient de dissymétrie n'est pas tout à fait constant, d'une part lorsque le débit varie, d'autre part suivant le sens de l'écoulement dans la galerie principale. De toute facon, la dissymétrie reste largement supérieure à 2 , valeur limite inférieure que nous avions admise a priori dans nos calculs, Done, les possibilités d'ouverture seront d'autant augmentées. Il est à noter qu'il aurait été possible d'obtenir une dissymétrie un peu plus grande en rapprochant la paroi verticale de son orifice, face i l'étranglement, mais on aurait eu alors un 《étranglement long \$ qui aurait prolongẻ d'autant le cheminement des ondes de coup de bétier.

\section{$1^{\circ}$ Ouvertures permises par la cheminée d'équilibre et le puits anti-bélier}

Ainsi que nous l'avons dit, les conditions d'ouverture sont beaucoup moins définies que les conditions de fcrmeture et on peut admettre une certaine adaptation de l'exploitation. Nous les avons néanmoins définies dans quelques cas types:

a) OUVerture Maximum DE $x$ A $100 \%$ ^ PARTIR du NIVeau statioue minimum 466. - Le calcul par le procédé rétrograde (sans tenir compte du puits antibélier) nous avait donné une ouverture maximum de $67 \%$ à $100 \%$. Nous avons refait la même épure en tenant compte du puits

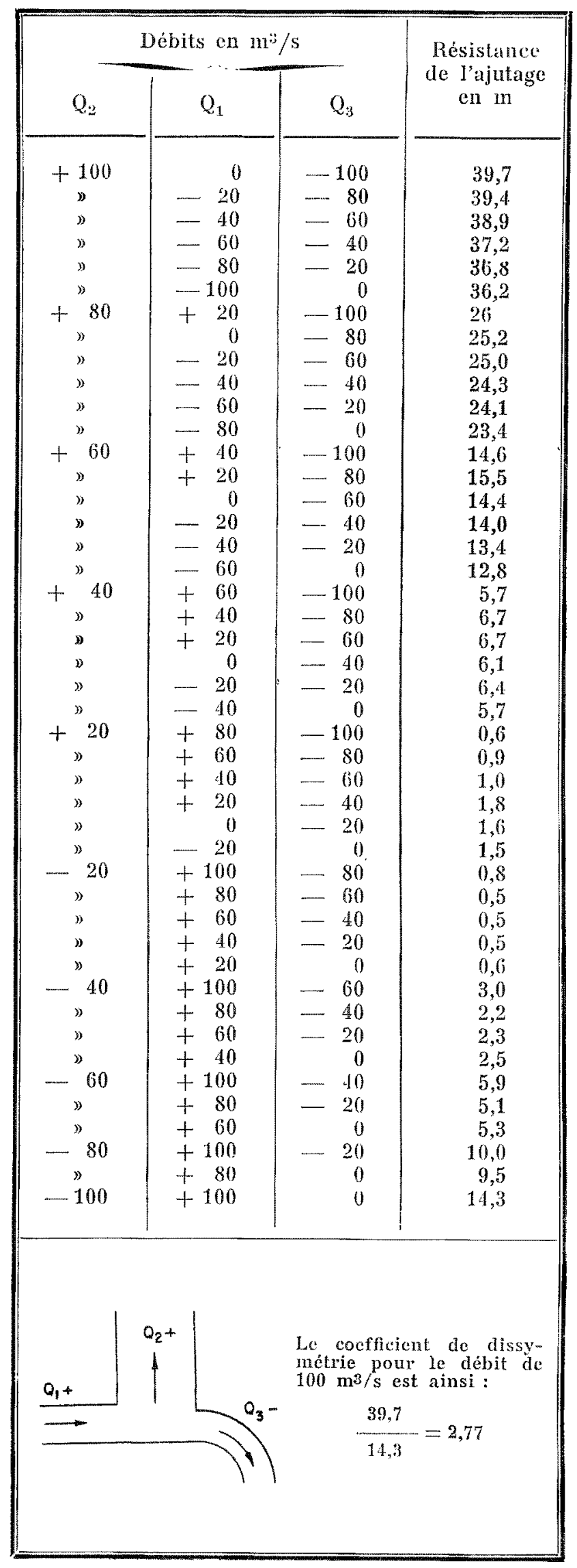


antibélier par la méthode habituelle indirecte (épure $n^{\circ} 2$ ); elle montre que l'influence du puits sur la cheminée d'équilibre est très faible et qu'elle agit dans ce cas particulier dans un sens plutôt favorable. On voit donc que l'ouverture est possible et que les oscillations dans le puits anlibéliex restent dans des limites acceptables et, en particulier, ne découvrent pas la calotle de la galerie. Il sera donc toujours possible de mettre en marche, sans précaulions spéciales, lc dernier groupe (ouverture de 75 à $100 \%$ ) quand on est en régime permanent.

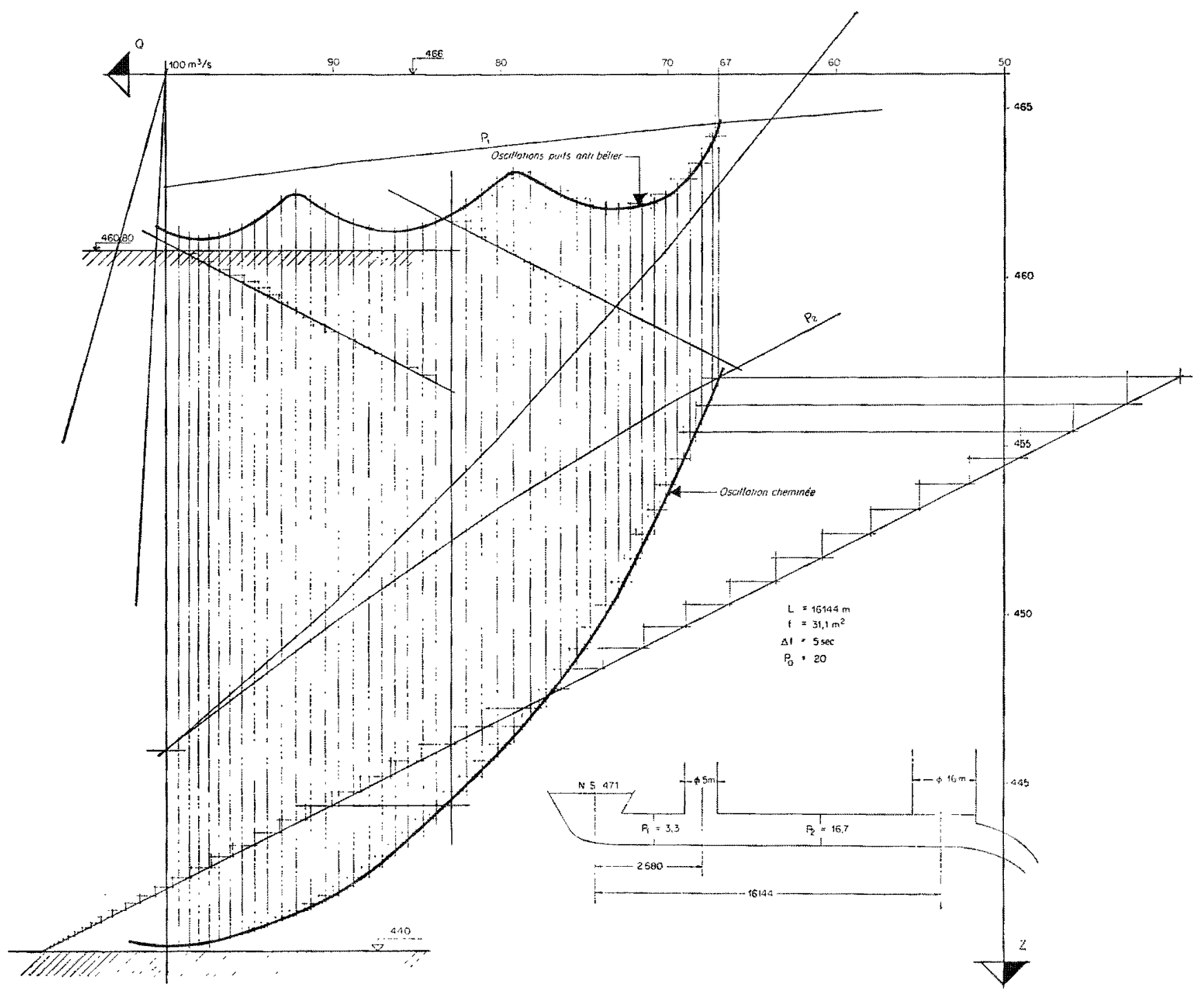

EPune 2. - Ouverture de 67 à $100 \%$.

b) OUverture de 0 A $50 \%$ a PARTIR DU NIveAu STATIQUe MINIMUM 466 . - Nous avons vérifié par une épure que la cheminée permettait la prise en charge immédiate de la moitié des. groupes en régime stable, la retenue étant au niveau statique minimum 466 et en tenant compte, bien entendu, du puits antibélier. La cote minimum de l'eau, au cours de cette manceuvre, atteint $444,60 \mathrm{~m}$, soit $4,60 \mathrm{~m}$ au-dessus du fond; il reste donc une marge de sécurité confortable; cependant, il est à remarquer que cette marge est bien moins importante dans le puits antibélier puisque, au cours des trois premières oscillations, le niveau dans ce puits descend presque jusqu'à la cote de la calotte de la galerie. Nous ne pensons pas d'ailleurs que cela soit un inconvénient rédhibitoire. L'ouverture maximum possible permise par la chemince d'équilibre serait de 0 à $68 \%$ (fig. 4).

c) Réouverture APRÈS DFCLENCHEMENT, - II est souvent intéressant, après un dóclenchement 
intempestif, de pouvoir recoupler au moins une partic de la puissance le plus tôt possible. Nous avons examiné, à cet égard, quelles étaient les possibilités de l'ouvrage projeté. Le problème peut se résoudre très facilement; traçons, en effet, pour le niveau statique minimum (cas le plus défavorable), l'épure de déclenchement complet et, à partir du niveau minimum dans la cheminée, les épures d'ouverture par le procédé rétrograde correspondant à différentes ouvertures finales. Une mancuvre donnée pourra être exécutée si l'épure d'ouverture reste à droite de l'épure de fermeture ou si elle lui est tangente; elle ne pourra être exécutée si elles se coupent. L'épure 3 donne le résultat; elle a été faite, pour simplifier, sans tenir compte du puits antibélier, les épures précédentes nous ayant montré, en effet, que celui-ci a très peu d'influence

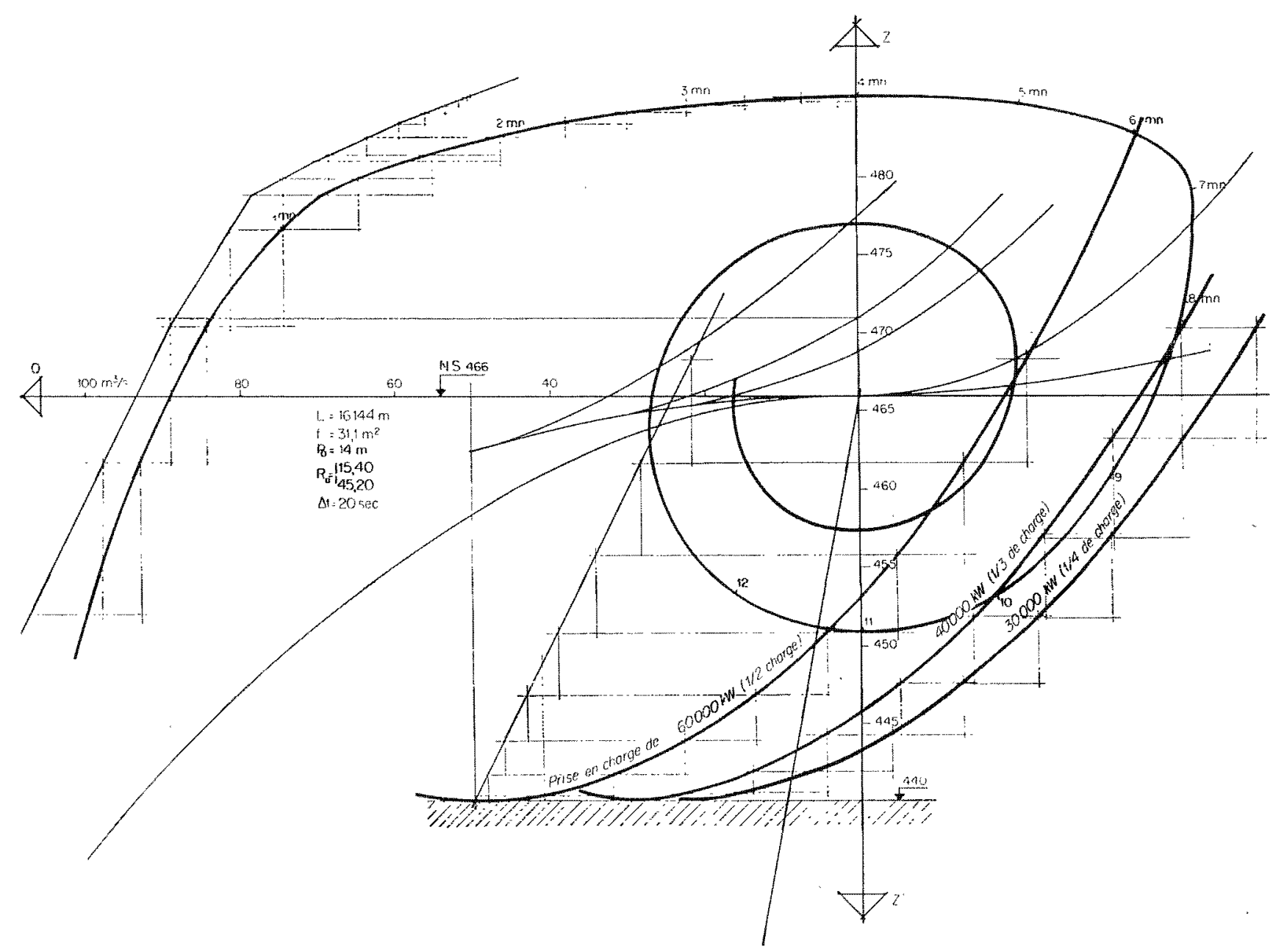

Epune 3. - Ouverture après déclenchement.

sur les oscillations dans la cheminée d'équilibre. On voit qu'on peut toujours mettre en charge le premier groupe (ouverture de 0 à $25 \mathrm{~m}^{3}$ ), mais qu'on ne peut mettre en charge deux groupes immédiatement (ouverture de 0 à $50 \mathrm{~m}^{3}$ ) qu'à condition de commencer l'opération avant que l'eau ait atteint son niveau maximum (première montée) ou après que l'eau ait atteint son niveau minimum (remontées suivantes); autrement dit, on peut toujours coupler à demi-charge après un déclenchement quand l'eau remonte dans la cheminée d'équilibre.

d) Ouverture totale en 8 mn. - Ces conditions d'ouverture avaient été admises après enquête auprès de l'exploitation. Nous avons vérifié que la cheminée d'équilibre permettait la manœuvre dans le cas précis suivant : ouvertures successives de chaque groupe en $1 \mathrm{mn}$ avee un intervalle de $1 \mathrm{mn} 20 \mathrm{~s}$ entre chaque manouvre. Nous avons donc fait une épure en prenant 
le niveau slatique de cote minimum 466 . Le niveau minimum de l'eau au cours de l'oscillation est atteint $10 \mathrm{mn}$ après l'ouverture du premier groupe et sa cote est $441,60 \mathrm{~m}$, soit encore $1,60 \mathrm{~m}$ au-dessus du fond de la cheminée.

On peut imaginer également d'autres manœuvres d'ouverture des quatre groupes en $8 \mathrm{~mm}$, par exemple :

- Ouverture linéaire en $8 \mathrm{mn}$ des quatre groupes; cette mancuvre est trop progressive et utilise très mal l'étranglement; elle est très défavorable. Néanmoins, le niveau minimum atteint (pour le niveau statique 466) est encore 440, soit exactement le fond de la cheminée.

- Ouverture en $1 \mathrm{mn}$ de deux groupes et, en même temps, ouverture linéaire en $8 \mathrm{mn}$ des deux autres groupes. Le minimum est atteint au bout de $4 \mathrm{mn}$ à la cote 442 , soit $2 \mathrm{~m}$ au-dessus du fond pour une mancuvre effectuée évidemment à partir du niveau statique minimum 466 ; cette manceuvre est bien plus favorable que la précédente, l'étranglement étant utilisé largement, surtout au début.

On pourrait certainement imaginer d'autres mancuvres en $8 \mathrm{mn}$, ulilisant encore mieux les possibilités de la cheminée; de toute façon, il résulte de ces études que l'opération est possible, mais qu'il serait bon que l'agent chargé des manouvres ait devant les yeux un appareil lui permettant de suivre facilement les oscillations du plan d'eau dans la cheminée d'équilibre (et spécialement leur vitesse), et, d'autre part, qu'il sache que la cheminée est mieux utilisée au cours de mancuvres saccadées (toutes proportions gardées, bien entendu) qu'au cours de manouvres progressives.

e) Ouverture pour $\mathrm{QH}=\mathrm{CSTE}$. - Il est pospossible que l'usine de Randens soit momentanément appelée à être utilisée en réseau séparé; son régulateur serait alors appelé à régler le débit à puissance constante, si on suppose le rendement constant; cela correspond au débil $\mathrm{QH}=\mathrm{Cste}$. Nous avons fait une épure relative à une ouverture de 0 à $50 \%$ en faisant $\mathrm{QH}=\mathrm{C}$ te par la méthode que nous avons exposée dans le numéro $3 / 1951$ de la Houille Blanche épure 4). Le plan d'eau est abaissé sur la manouvre classique de $2,80 \mathrm{~m}$.

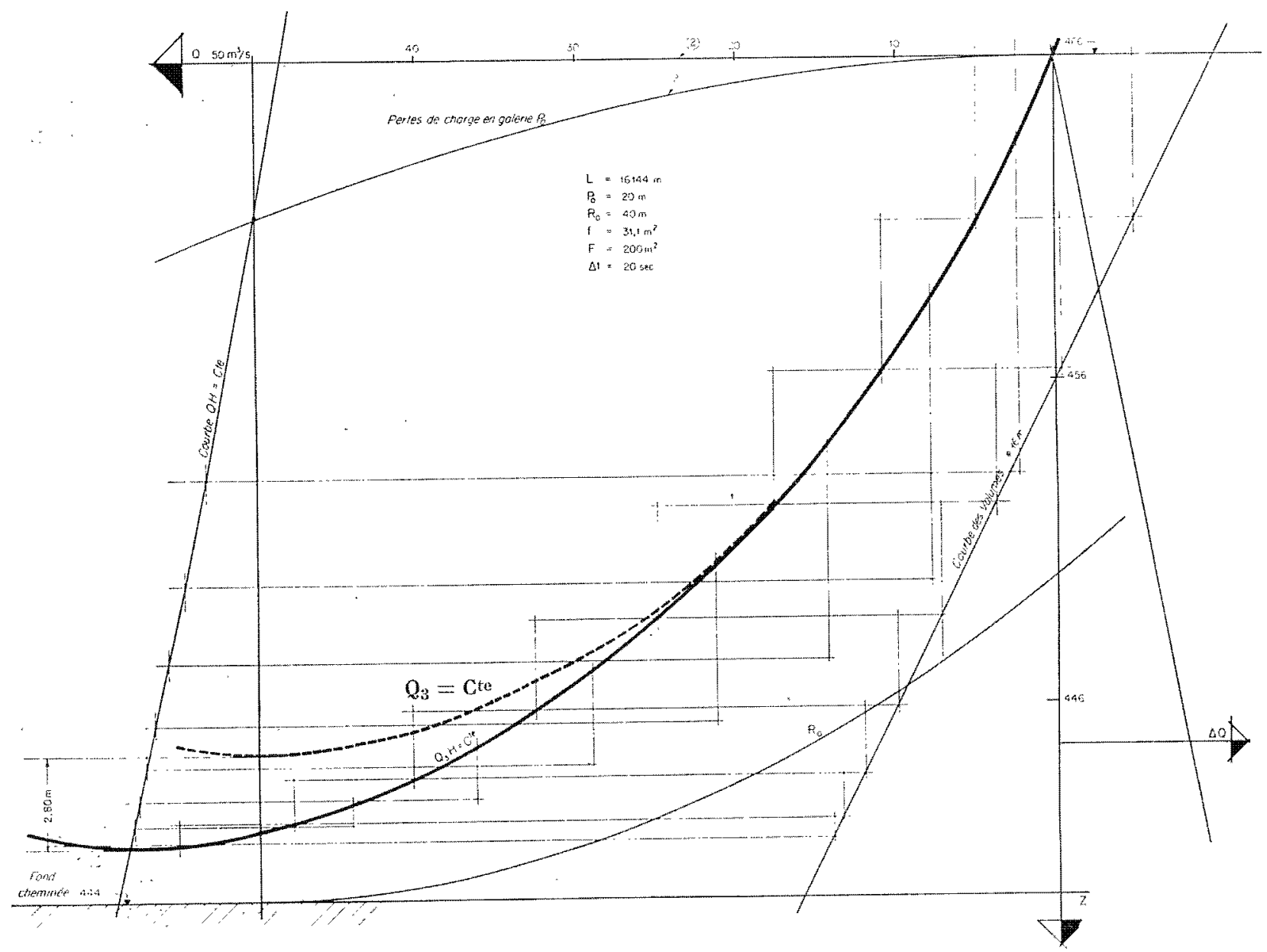

Epune 4. - Ouverture de 0 it 50 \% ave $\mathrm{OH}=\mathrm{Cte}$. 
f) 'TEMps pendan't Lequel le niveau Reste EN-DEsSOUS DU PIìge A BULLES. - Nous avons déterminé, à l'aide du procédé rétrograde, le temps pendant lequel le niveau reste en-dessous du piège à bulles, ce qui nous a servi à dimensionner le réservoir correspondant. L'application du procédé est immédiate et a été également expliquée dans nos études précédentes. Le temps maximum durant lequel le niveau risque de rester en dessous du désaérateur est de $5 \mathrm{mn}$.

\section{$2^{\circ}$ Etude des mancurres de fermeture et des surpressions en galerie}

a) Epure de Bergeron. - Les ondes de surpression provoquées par l'étranglement ne permettaient pas de suivre, dans une épure d'oscillation en masse, la surpression en chacun des points de la galerie. La connaissance de son. évolution en chaque point nous paraissait cependant particulièrement intéressante, et, dans ce but, nous avons fait une épure de BERGERon en nous efforçant de nous rapprocher le plus possible des conditions réelles. Cette épure se rapporte à un déclenchement à pleine puissance en $3 \mathrm{~s}$. Nous avons été amenés cependant à modifier un peu la méthode Bergeron classique en utilisant, dans cette épure, la courbe des volumes des épures classiques pour calculer les montées d'eau dans la cheminée d'équilibre et dans le puits antibélier; cette petite modification, qui ne change évidemment rien au principe bien connu de la méthode de Bergeron, est simplement un artifice qui peut être utile dans l'étude des oscillations du plan d'eau dans une cheminée d'équilibre et qui nous a permis de gagner du temps et de la précision dans cette épure. Nous allons exposer succinctement ce procédé.

Considérons (figures 12 et 13 ) un puits de section $\mathbf{F}$ branché sur une galerie d'amenée:

Soit $Q_{t}$ et $Z_{t}$ ses caractéristiques débit-niveau à l'instant $t$, représenté par le point figuratif $A_{t}$.

Cherchons le point figuratif $B_{t+\Delta t} d \mathbf{u}$ puits à l'instant $t+\Delta t$; la méthode de Bergeron

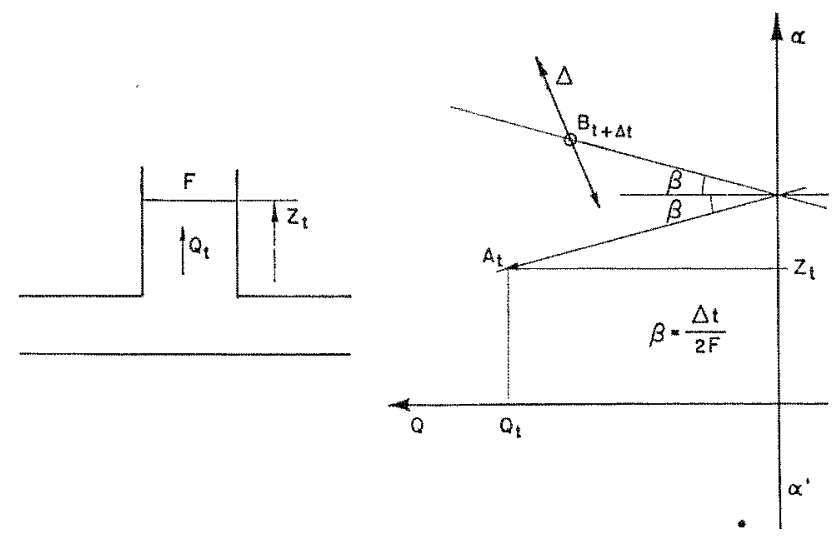

Frg. 12

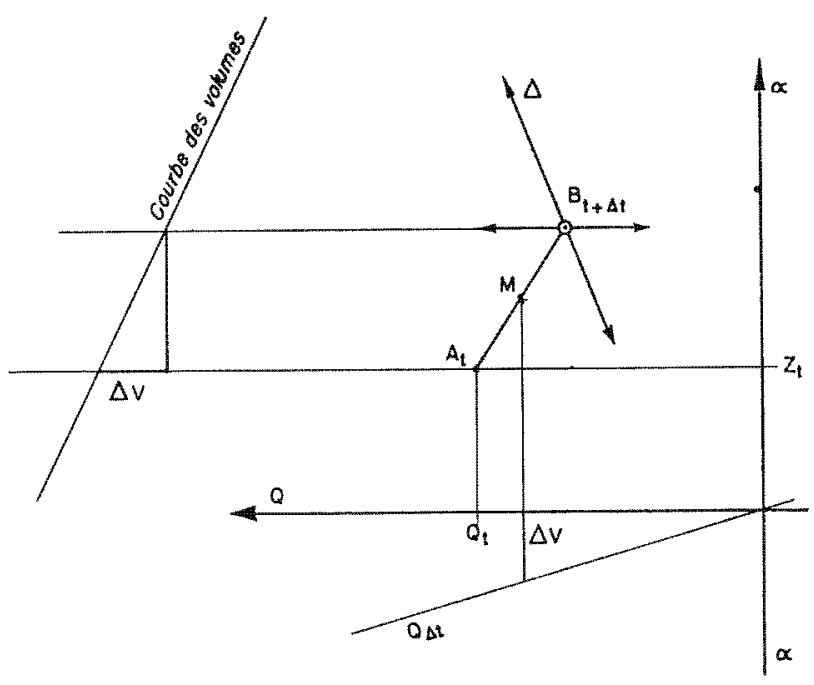

Fig. 13

nous permet de trouver un premier lieu de $\mathrm{B}_{t+\Delta t}$, la droite $\Delta$ déduite de considérations sur les ondes, trop connue pour que nous nous y arrêtions. Pour évaluer la montée du niveau pendant cet intervalle, M. Bergeron propose d'utiliser deux droites de pente $\beta=\Delta t /(2 \mathrm{~F})$, se réfléchissant sur l'axe des $Z$ (figure 12 ).

L'intersection de la deuxième droite avec $\Delta$ donne le point $B_{t+\Delta t}$; en effet, il est facile de calculer que la montée de l'eau dans le puits ainsi défini est $\left(\mathrm{Q}_{t}+\mathrm{Q}_{t+\Delta t}\right) \Delta t /(2 \mathrm{~F})$. Théoriquement, donc, la méthode est rigoureuse pour des $\Delta t$ petits, mais dans le cas des épures que nous avons faites, la pente $\beta$ était si faible qu'il était très difficile de tracer d'une manière précise ces deux droites, surtout en début d'épure, quand elles traversent presque entièrement le graphique; d'autre part, à chaque changement de section de la cheminée d'équilibre (et il y en a trois dans le cas de RANDENs), il fallait changer de pente $\beta$, ce qui était encore une complication. Nous avons done tout simplement adjoint, à notre épure de BERGERoN, la courbe des volumes de la méthode Schoklitsch (fig. 13), ainsi que la droite des $Q_{\Delta t}$. On apprécie à l'avance la position $d u$ milieu $M$ du segment $A B$, qui a pour abseisse $\frac{Q_{t}+Q_{t+\Delta t}}{2}$. A l'aide de la courbe des volumes, on a la montée d'eau résultante durant cet intervalle $\left(\frac{\mathrm{Q}_{t}+\mathrm{Q}_{t+\Delta t}}{2 \mathrm{~F}}\right) \Delta t$. Les deux méthodes aboutissent donc au même résultat. Evidemment, dans la deuxième, il faut apprécier à l'avance le milieu $M$ du segment $A B$, ce qui n'est pas absolument rigoureux, mais pratiquement, dans nos épures faites avec des intervalles de temps très petits à l'échelle du phénomène $(\Delta t=6 \mathrm{~s}$ pour une période de $16 \mathrm{mn})$, cette méthode était nettement plus pré- 
cise que la première et surtout les épures étaient beaucoup plus rapides. L'épure $n^{\circ}$ I a été faile suivant ce procédé.

Les hypothèses sont les suivantes:

$$
\begin{array}{lll}
f=31,1 & \mathrm{Q}=100 & \text { N. } \mathrm{S} .=471 \\
\mathrm{R}_{0} 15 \mathrm{~m} & \mathrm{P}_{0}=14 \mathrm{~m} &
\end{array}
$$

Temps de fermeture: $3 \mathrm{~s}=\%$

Intervalle de temps: $\Delta t=6 \mathrm{~s}=2 \tau$.

Vitesse des ondes dans la galerie $=900 \mathrm{~m} / \mathrm{s}$.

Longueur de la galerie $=16.200 \mathrm{~m}$.

Distance du puits antibélier à la prise d'eau $=2.700 \mathrm{~m}$.

Remarove. - Le temps de fermeture et la vitesse des ondes ne sont pas tout à fait conformes à la réalité, mais leurs valeurs ont été choisies pour avoir à modifier le moins possible la longueur de la galerie $(16.200 \mathrm{~m}$ au lieu de 16.144) et la distance du puits antibélier à la prise d'eau $(2.700 \mathrm{~m}$ au lieu de $2.680 \mathrm{~m})$, en effet, ces longueurs étant justement des multiples entiers du produit $3 \times 9$. Cet artifice, qui modifie très peu les résultats, tout en gardant leur aspect qualitatif, simplifie énormément l'épure de Bergeron, qui, sans cela, deviendrait tellement compliquée qu'elle serait pratiquement infaisable.

Par mesure de précaution, nous avons super- posé à celte épure une épure d'oscillation en masse en partant évidemment des mêmes hypothèses. On voit que pour la montée de l'eau dans la cheminée d'équilibre, la superposition est bonne, puisque les cotes sont respectivement $488 \mathrm{~m}$ et $488,20 \mathrm{~m}$. Nous rappelons que la cheminée d'équilibre a été calculée pour le niveau maximum théorique de 488 ; on voit donc que les simplifications dont nous avons parlé plus haut n'ont pratiquement pas modifié le résultat final.

b) Surpression en Galenie. - Cette épure nous a permis de faire le graphique des surpressions en différents points de la galerie, en fonction du temps, au cours d'un déclenchement; les résultats sont transcrits sur le graphique de la figure 14. Le point $\mathrm{P}$ est situé à $2.700 \mathrm{~m}$ à l'amont de la cheminée d'équilibre, $H$ est à $7.100 \mathrm{~m}$ et $\mathrm{K}$ est à $9.800 \mathrm{~m}$. On suit d'une manière curieuse le passage en chacun de ces points du train d'ondes provoquées par la brusque utilisation de l'étranglement, et on apprécie ainsi combien le phénomène est important. Une remarque s'impose également au sujet de la protection créée par le puits antibélier du côté aval. Théoriquement, en effet, l'onde se fait sentir intégralement jusqu'à une distance égale à $a \mathrm{~T} / 2$ de ce puits, la fermeture des turbines étant supposée linéaire; la surpression sous la cheminée a, en fonction du temps, une allure parabolique jusqu'au maximum atteint une fois

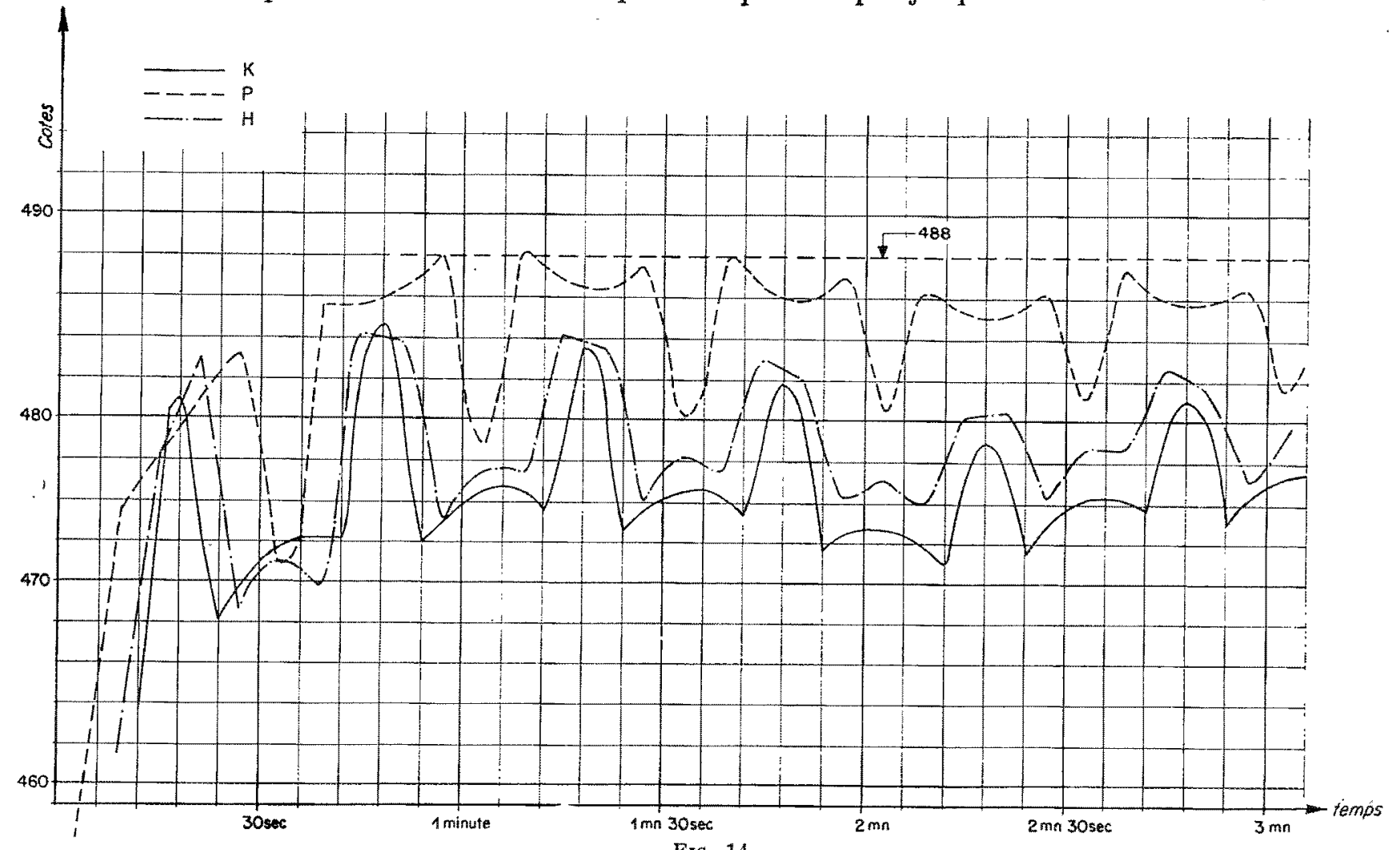

FIG. 14 
la fermeture achevée. Elle se propage sous cette forme le long de la galerie; la dépression, renvoyée par la surface libre du reniflard, est représentée par la même parabole de signe opposé. L'allure des surpressions maxima, en un point donné, s'en déduit facilement si l'on remarque que la surpression maximum, en un point, est atteinte quand l'onde directe aboutit à son maximum au point donné. Le calcul est facile et donne, en un point d'abscisse réduite $x$ pour les surpressions :

$$
y_{m}=4 x-4 x^{2}
$$

$y$ représentant la surpression réduite, e'est-àdire rapportée à la valeur de la perte de chargo à travers l'étranglement,

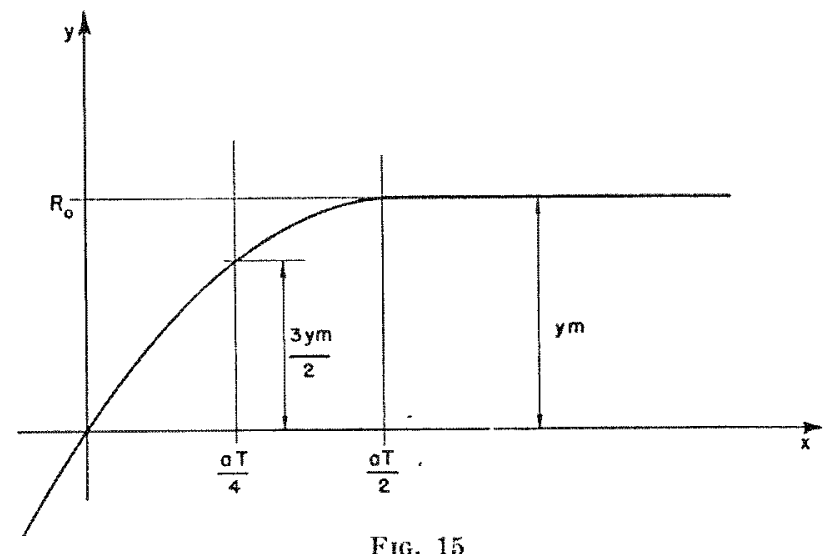

$x$ l'abscisse réduite, esest-à-dire rapportće à la distance $a \mathrm{~T}$.

Il en résulte que la surpression due aux coups de bélier est tout de suite relativement importante; ainsi, figure 15, à la distance $a \mathrm{~T} / 4$ à l'aval du puits antibélier, c'est-à-dire à environ $1.000 \mathrm{~m}$ dans le cas de Randens, la surpression due aux coups de bélier est encore égale à $3 / 4$ de $y_{m}$, c'est-à-dire à plus de $11 \mathrm{~m}$, et non à $y_{m} / 2$, soit $7,50 \mathrm{~m}$, comme on aurait tendance à le croire à première vue.

Nous aurons done à tenir comple, en un point de la galerie, de deux surpressions de nature bien différente, bien qu'en définitive toutes deux soient provoquées par des ondes:

- la première, due au coup de bélier de l'étranglement, dont la répartition en fonction de la distance vient d'être étudiée,

- la seconde, due à la montée maximum du niveau dans la cheminée répartie linéairement en fonction de la distance, au moins si la section de la galerie est constante.

Il y a évidemment lieu de comparer ces deux maxima et de les équilibrer par un choix judicieux de la puissance de l'étranglement. Il serait d'ailleurs souhaitable et logique que la surpression du coup de bélier soit inférieure à la surpression d'oscillation en masse, étant donné ses effets dynamiques néfastes. A Randens, cepen- dant, cette condition n'est pas tout à fait respectée, puisque, dans la partie de galerie située entre Notre-Dame-de-Briancon et l'Etrat, les surpressions dues au coup de bélier sont légèrement supérieures à celles résultant de l'oscillation en masse. Pour satisfaire complètement à cette condition, il aurait fallu placer le reniflard a. Notre-Dame-de-Briançon, mais alor's ses dimensions auraient été considérablement plus grandes.

c) Condition de Fermeture la plus défayoRABLE. - Il est bien connu que la fermeture instantanée n'est pas la condition la plus défavorable dans les cheminées à étranglement, l'écart pouvant être d'autant plus grand que l'étranglement est plus efficace.

Nous avons donc appliqué une méthode proposée par les ingénieurs du Laboratoire Dauphinois d'Hydraulique, à la suite de notre article du numéro $3 / 1951$ de la Houille Blanche, qui permet de trouver directement le cas de fermeture le plus défavorable; cette ouverture n'est d'ailleurs pas, en général, linéaire.

L'écart, avec la fermeture instantanée, est, en réalité, faible, mais ce procédé peut éventuellement présenter un grand intérêt si l'étranglement est plus puissant. Dans le cas de Randens, la fermeture la plus défavorable se fait en $1 \mathrm{mn} 10 \mathrm{~s}$; il est à remarquer qu'elle n'est pas linéaire; la surélévation du niveau maximum de l'eau au cours d'un déclenchement est inférieure à $20 \mathrm{~cm}$.

d) DEscente CONSÉCUTIVE A LA PREMIÉre MONTÉE. - VIDANGE DES CHAMBRES D'EXPANSION. On suppose, tant à la montée qu'à la descente, que le niveau, dans les chambres d'expansion et

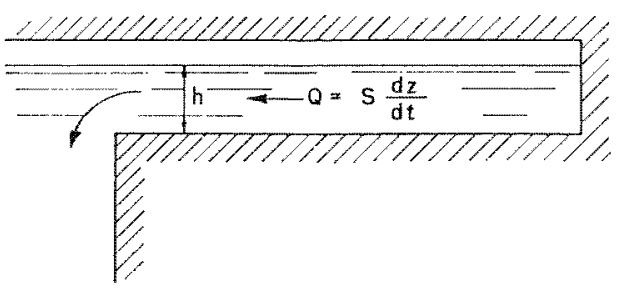

Fig. 16

dans le puits, reste horizontal. Cette hypothèse n'est qu'approximative, car il faut évidemment que la hauteur d'eau, à l'entrée de la chambre, soit suffisante pour permettre, au moins en régime dénoyé, d'écouler tout le débit de remplissage de la chambre, soit $\mathrm{S} d z / d t$ (fig. 16).

Il est très facile de voir que cette influence est relativement faible dans le cas de cette cheminée, en raison de la section du puits central. Nous l'avons examiné, à la descente, de la façon suivante :

On relève, sur une épure classique, la courbe $\mathrm{Q}(t)$ et $h(t)$ pour chacune des chambres d'ex- 
pansion et on trace la courbe des hauteurs critiques correspondant aux débits $Q$ (fig. 17).

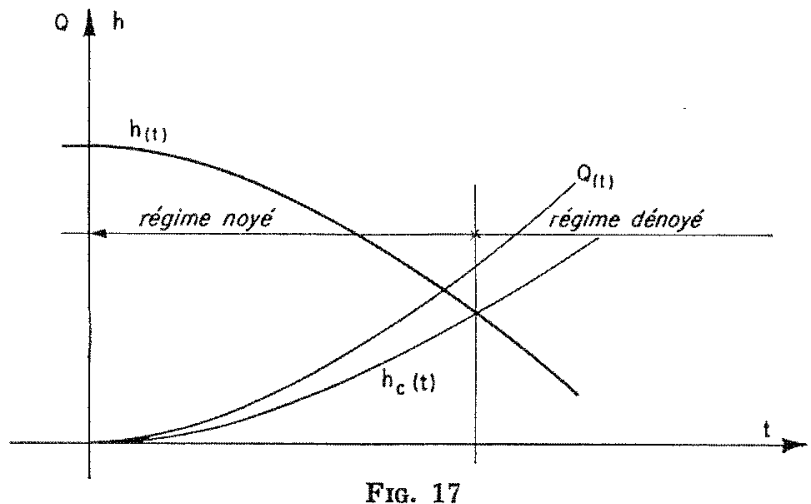

Tant que la hauteur dans la chambre est supérieure à la hauteur critique, le débit écoulé correspond sensiblement à ce que donnerait l'hypo- thèse du niveau constant et horizontal entre puits et chambre.

Au-delà, le débit des chambres est donné par la hauteur à l'aval des chambres d'expansion. Le niveau dans le puits évolue indépendamment du niveau dans les chambres.

Les courbes de la figure 18 , tracées en vraie grandeur, confirment que cet effet se produit juste à la fin de la vidange des chambres. Il est d'ailleurs favorable, puisqu'une partie de l'énergie potentielle des chambres est dissipée lors de la chute dans le puits.

Nous verrons, dans un article ultérieur, que ce phénomène peut prendre une importance beaucoup plus grande dans une cheminée à chambre d'expansion et puits de faible diamètre. Nous avons eu l'occasion de le vérifier en faisant fonctionner à pleine charge la cheminée de l'usine de Passy.

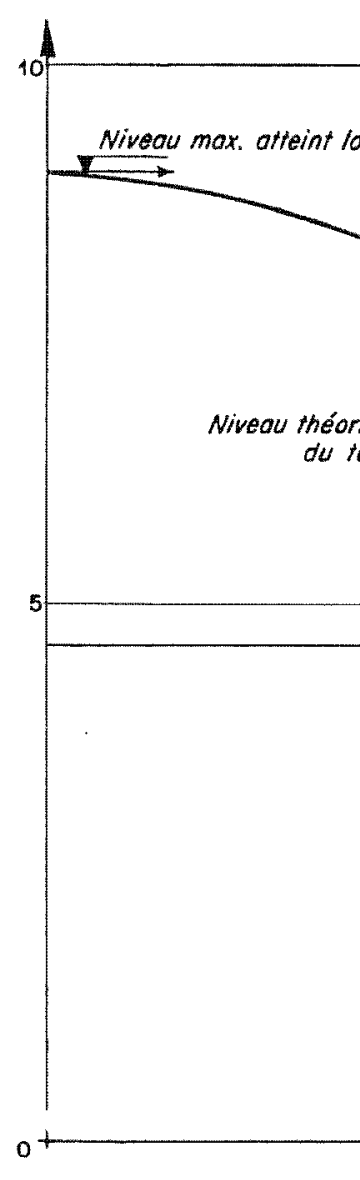

\section{Conclusion}

C'est sur ces différentes études que nous avons basé notre projet de cheminée d'équilibre. Etant donné les caractéristiques importantes de l'ouvrage, il a été nécessaire de déborder un peu le cadre des études classiques et d'approfondir no-
Déclenchement a pleine charge $0=100 \mathrm{~m}^{3} / \mathrm{sec}$

N.S. 471

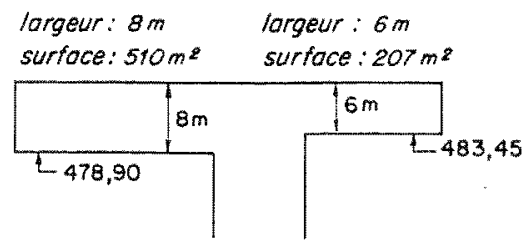

- Courbe des houteurs critiques pour le dóbit théorique

Niveau petite chambre

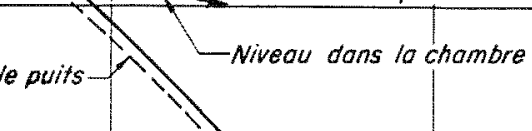

$\frac{\text { Nivsou, grande chambre }}{3}$

Fir. 18

tablement certaines questions relatives aux surpressions provoquées par l'étranglement.

Il y a lieu, pensons-nous, de porter une attention spéciale sur ce sujet quand la perte de charge créée par l'étranglement est un peu forte, même si la galerie est assez courte, car on introduit ainsi des phénomènes dynamiques, dont il y a lieu de préciser l'ampleur. 